Available online at www.sciencedirect.com

science $\mathcal{W}$ Direct.

\title{
Patterning with block copolymer thin films
}

\author{
Rachel A. Segalman* \\ Department of Chemical Engineering, University of California at Berkeley, Division of Materials Science, \\ Lawrence Berkeley Labs, 201D Gilman Hall, Berkeley, CA 94720-1462, USA
}

Received 13 October 2004; accepted 10 December 2004

\begin{abstract}
The nanometer-scale architectures in thin films of self-assembling block copolymers have inspired a variety of new applications. For example, the uniformly sized and shaped nanodomains formed in the films have been used for nanolithography, nanoparticle synthesis, and high-density information storage media. Imperative to all of these applications, however, is a high degree of control over orientation of the nanodomains relative to the surface of the film as well as control over order in the plane of the film. Induced fields including electric, shear, and surface fields have been demonstrated to influence orientation. Both heteroepitaxy and graphoepitaxy can induce positional order on the nanodomains in the plane of the film. This article will briefly review a variety of mechanisms for gaining control over block copolymer order as well as many of the applications of these materials. Particular attention is paid to the potential of perfecting long-range two-dimensional order over a broader range of length scales and the extension of these concepts to functional materials and more complex architectures.
\end{abstract}

(C) 2005 Elsevier B.V. All rights reserved.

Keywords: Block copolymers; Nanopatterning; Thin films; Microphase separation; Polymer self-assembly; Block copolymer lithography

\section{Introduction}

Block copolymers have been suggested for many applications based principally on their ability to form regular nanometer-scale patterns. These self-assembled patterns have been considered as nanolithographic masks as well as templates for the further synthesis of inorganic or organic structures. Such applications are made possible by exploiting contrasts in chemical or physical properties that lead to differential etch rates or attractions to new materials. New applications in fuel cells, batteries, and optoelectronic devices rely on the inherent properties of the blocks. All of these uses depend on the extremely regular self-assembly of block copolymers over macroscopic distances. Frequently, however, the orientation of the nanodomains is not perfect or the grain size is insufficiently large. In these cases, much work has been done to control the self-assembly and orientation of block copolymers. This review will discuss many of these control techniques and also several of the targeted applications.

A block copolymer consists of two or more polymeric chains (blocks), which are chemically different and covalently attached to each other. In the melt, they are driven to segregate into a variety of ordered structures by the repulsion of the immiscible blocks, much as in the case of a blend of immiscible homopolymers. In this far simpler case of a blend of two homopolymers, A and B, the

* Tel.: +1 510642 7998; fax: +1 5106424778 .

E-mail address: segalman@berkeley.edu. 
phase behavior may be controlled by three experimental parameters: the degree of polymerization $(N)$, the composition $(f)$, and the A-B Flory-Huggins interaction parameter $(\chi)$. The Flory-Huggins equation describes approximately how these parameters affect the free energy of a blend:

$$
\frac{\Delta G_{\mathrm{mix}}}{k_{\mathrm{b}} T}=\frac{1}{N_{\mathrm{A}}} \ln \left(f_{\mathrm{A}}\right)+\frac{1}{N_{\mathrm{B}}} \ln \left(f_{\mathrm{B}}\right)+f_{\mathrm{A}} f_{\mathrm{B}} \chi
$$

The first two terms correspond to the configurational entropy of the system, and can be regulated via the polymerization chemistry to change the relative lengths of the chains and fractions of A versus $B$ polymer. In the third term of (1), $\chi$ is associated with the non-ideal penalty of A-B monomer contacts and is a function of both the chemistry of the molecules and temperature. In general,

$$
\chi=\frac{a}{T}+b
$$

where $a$ and $b$ are experimentally obtained constants for a given composition of a particular blend pair. Experimentally, $\chi$ can be controlled through temperature. Unlike macrophase separation in blends, the connectivity of the blocks in block copolymers prevents complete separation and instead the diblock copolymer chains organize to put the $\mathrm{A}$ and $\mathrm{B}$ portions on opposite sides of an interface. The equilibrium nanodomain structure must minimize unfavorable A-B contact without over-stretching the blocks [1]. The strength of segregation of the two blocks is proportional to $\chi N$. A symmetric diblock copolymer is predicted to disorder (or pass through its order-disorder temperature (ODT)) when $\chi N<10$. Below the ODT and when the volume fraction of block $\mathrm{A}\left(f_{\mathrm{A}}\right)$ is quite small, it forms spheres in a body-centered cubic (BCC) lattice surrounded by a matrix of $\mathrm{B}$. As $f_{\mathrm{A}}$ is increased towards 0.5 , the minority nanodomains will form first cylinders in a hexagonal lattice, then a bicontinuous double gyroid structure, and finally lamellae. The size and periodicity of the nanodomains are also functions of the polymer size $(N)$ and the segmental interactions $(\chi)$, as shown in Fig. 1 [1,2].

(a)

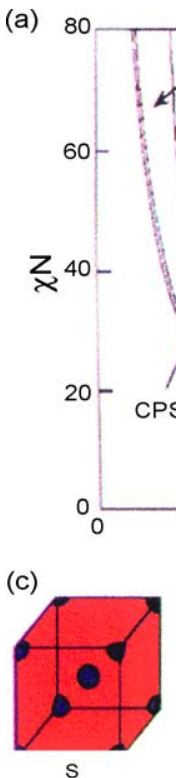

$\mathrm{s}$
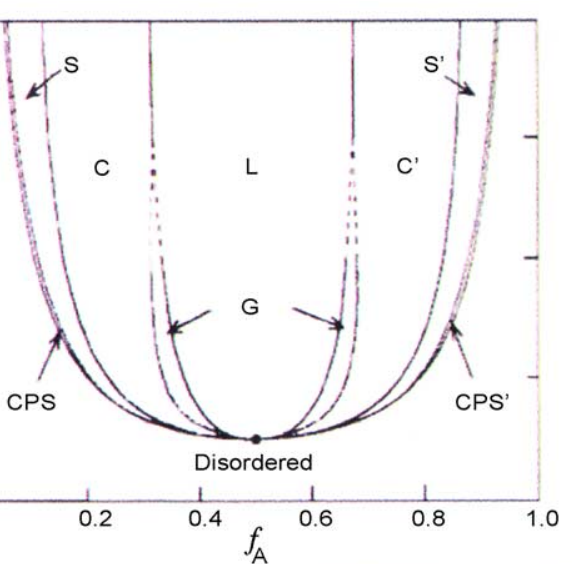

(b)
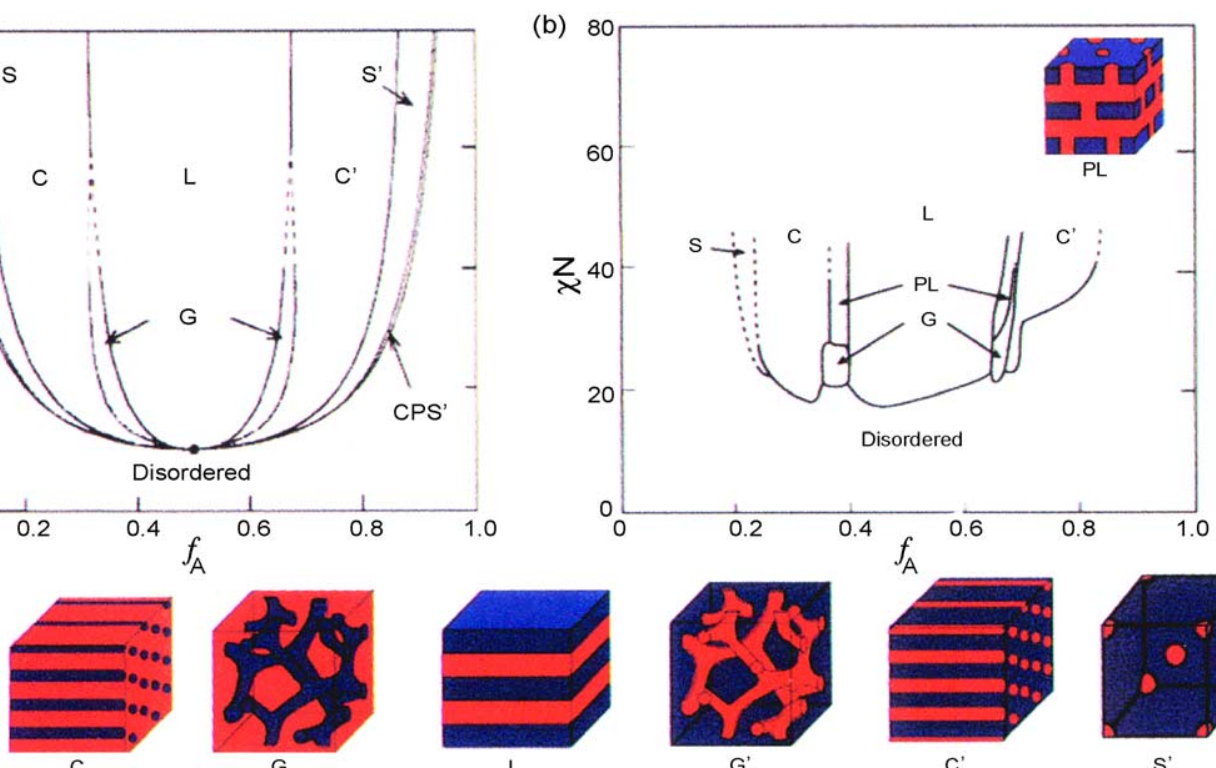

C

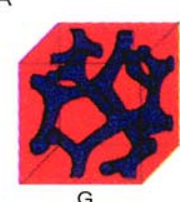

G

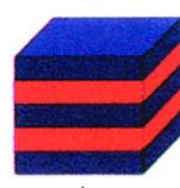

L

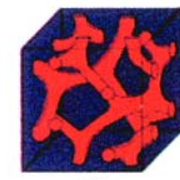

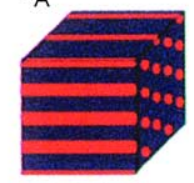

$C^{\prime}$

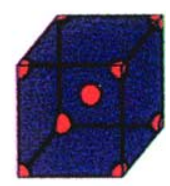

S'

Fig. 1. Diblock copolymers are predicted to self-assemble according to a phase diagram predicted by self-consistent mean field theory (a) and proven experimentally (b). A variety of constant-radius geometries are observed as a function of relative lengths of the two blocks (c). Reproduced with permission from Physics Today [2]. 

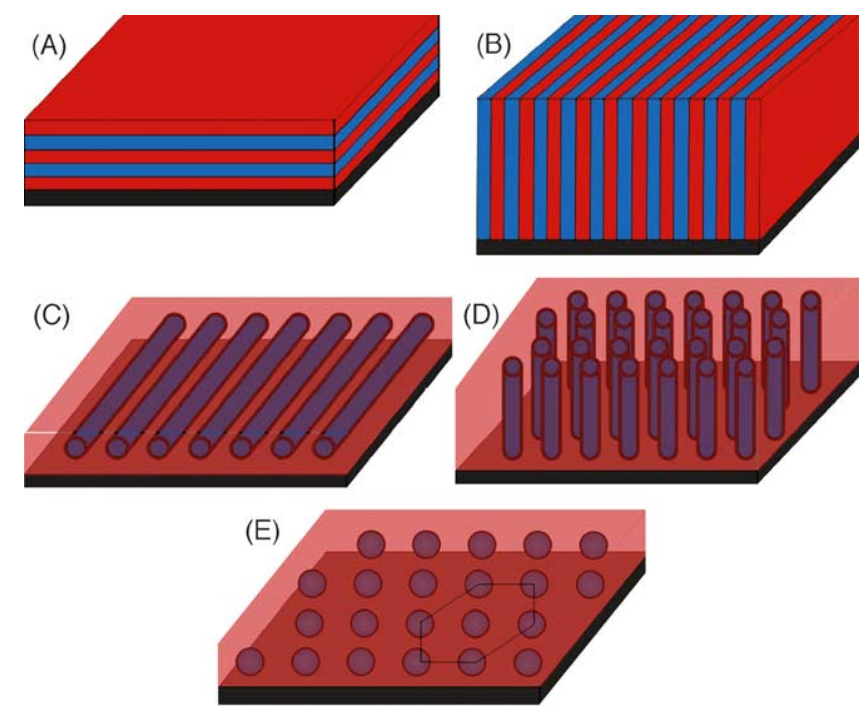

Fig. 2. When confined to a thin film, the orientation of block copolymer domains with respect to the substrate surface is crucial for many applications. (A) Lamellae lying parallel to the substrate, (B) lamellae aligned perpendicular, (C) cylinders lying parallel, (D) cylinders perpendicular, and (E) spheres. In the case of lamellae in the perpendicular orientation and cylinders in parallel, lines can be patterned if the persistence length of the structure can be controlled. In the case of upright cylinders and spheres, the grain size and perfection of the hexagonal array is of primary importance.

In the thin film state, the block copolymer nanodomain formation takes place relative to the surfaces of the film. As demonstrated in Fig. 2, the nanodomains tend to form with a particular orientation to the substrate surface. In the case of shapes with long axes (cylinders and lamellae), the orientation of the long axes with the surface is a major characteristic of the film. Cylinders lying parallel to the surface and lamellae standing perpendicular may each be of interest in the patterning of nanowires. Upright cylinders and spheres may be of interest in the patterning of hexagonal arrays for data storage. In this article, many the current and potential applications of block copolymer thin films will be discussed. While on a local scale, the nanodomains formed by the block copolymers are extraordinarily regular, it is difficult to control the order (meaning the orientation with the respect to the substrate and placement in space) on a more global scale. This review will begin by discussing many of the applications of block copolymer thin films, the control strategies and then conclude by looking forward to many unexplored systems and new applications.

\section{Applications}

Block copolymer thin films are of particular interest because of the possibility of obtaining twodimensional patterns with very high registry and regularity. In particular, they provide access to a length scale that is not available to traditional lithographic techniques. Although block copolymers have also made extensive inroads to bulk applications ranging from drug delivery to structural materials, this section will focus on applications of thin films. One focus of great interest has been in the patterning of microelectronics components on a length scale inaccessible by optical lithography. Many device structures are simply miniaturized descendents of current electronics. For instance, highdensity hard drives can be made by using the polymer nanodomains to pattern magnetic bits with a significantly greater number of bits per unit area than the current optical lithographically patterned drives. Other applications are wholly new and based on the ability to template the block copolymer 
directly. For instance, poly(ethylene oxide) (PEO) containing block copolymers may be very useful as the electrolyte layer of a battery or fuel cell, particularly if the electrolyte channel can be patterned in with a particular orientation. Furthermore, as the synthesis of conducting polymers and metalcontaining block copolymers matures, the ability to pattern materials on the nanometer length scale extends into yet another new range of applications. In this section, applications will be broken down into two categories: top-down and bottom-up techniques. Top-down techniques, in general, refer to the traditional methods of the semiconductor industry in which pattern transfer from the block copolymer is used to pattern an underlying substrate. In bottom-up techniques, the active components are directly self-assembled.

\subsection{Top-down fabrication}

Traditional microelectronics fabrication has relied on approaches involving the direct patterning of subsequent layers. Trends to smaller feature sizes, as exemplified by Moore's law, with inherently decreased diffusion distances and increased surface/volume ratios are rapidly approaching the limits of traditional optical lithography, approximately $100 \mathrm{~nm}$. While several alternatives have been introduced, many are prohibitively expensive. All of the following current lithography techniques rely on the same basic principle: the exposure of a material to electromagnetic radiation which chemically alters the exposed regions to make them either more or less soluble. The image is then developed into a relief structure which can then be transferred into another layer either by etching or by the subsequent deposition of material. Optical lithography is simply the exposure of some of the resist to ultraviolet light through a mask which shields the unexposed portions. This method, however, is limited by the wavelength of light (submicron). Electron beam lithography is capable of far smaller features, but each feature must be written into the surface individually, rather than the massive parallel exposure that occurs in optical lithography. Interference lithography employs the interactions between coherent laser beams to directly pattern the resist on a submicron length scale. Zone-plate array lithography accomplishes a similar result using an array of Fresnel zone plates to focus an X-ray source. Both of these techniques, however, require moderately expensive, new equipment. Nanoimprinting, which will be discussed in more detail later in this review, is an interesting route to repeatedly and relatively inexpensively pattern surfaces. This technique requires a master patterned by some alternate route to serve as a model. Self-assembled block copolymers as lithographic masks offer an interesting, potentially inexpensive alternative. In this case, the block copolymer thin film is used as a sacrificial layer to transfer a dense set of nanoscale patterns into the desired material. The goal is generally to achieve highly uniform structures with high aspect ratios for application in a variety of devices from capacitors and biochips to quantum dot arrays and transistors.

\subsubsection{Nanolithography}

Self-assembling routes to regular patterns on length scales less than $100 \mathrm{~nm}$ are attractive due to their relative ease of implementation and low cost. Nanometer-scale silicon oxide patterns are potentially useful as dynamic access memory stack capacitors, while magnetic dots may form useful hard drives. For each of these applications, the block copolymer pattern must be transferred to the appropriate material by either etching an underlying substrate or depositing the new material on top. Harrison et al. have recently published a comprehensive review of the transfer of patterns targeted to those familiar with traditional lithographic techniques [3].

Park et al. first demonstrated the use of block copolymer films as etch masks to transfer patterns to an underlying semiconductor substrate [4]. As shown in Fig. 3, the film can be used as a positive mask by removing the minority block (in this case by selective ozonation which attacks the double bonds in 


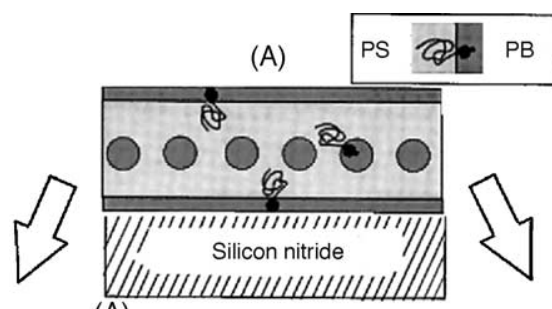

(B)

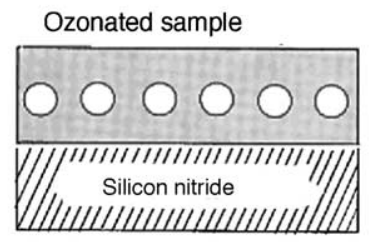

$\operatorname{RIE}\left(\mathrm{CF}_{4}\right.$ or $\left.\mathrm{CF}_{4} / \mathrm{O}\right)$
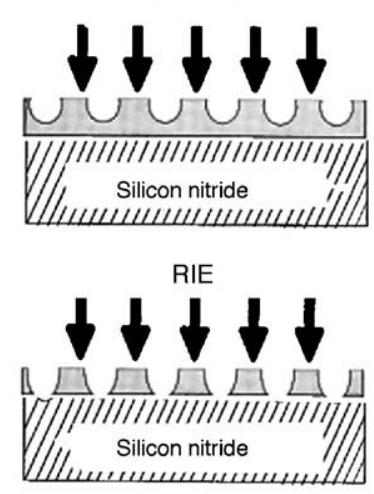

(C)

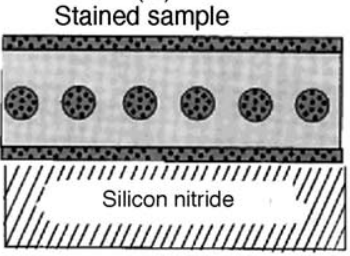

$\mathrm{RIE}\left(\mathrm{CF}_{4} / \mathrm{O}\right)$

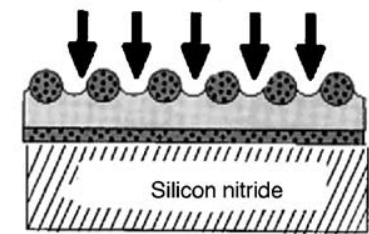

RIE

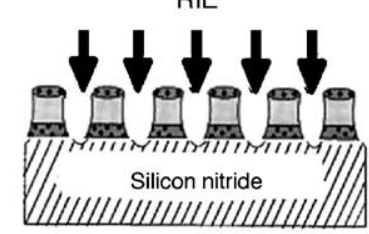

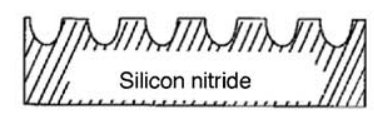
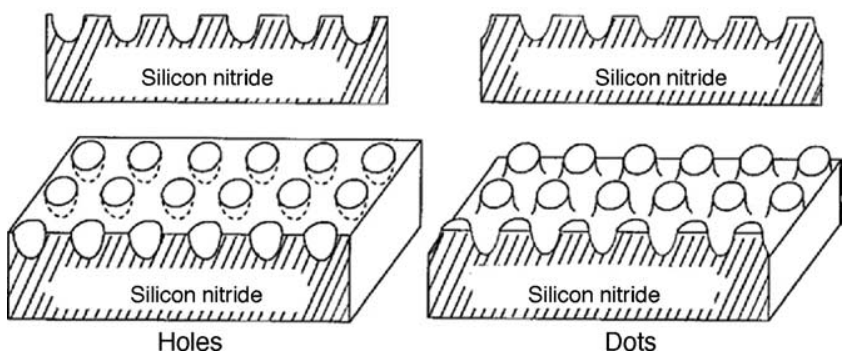

Fig. 3. Processing scheme for block copolymer lithography. The sequential removal of one component and further etching of the sample replicate patterns on the order of tens of nanometers to a SiN surface (from [4]). Route B demonstrates a positive resist, while route $\mathrm{C}$ demonstrates a negative one. Reproduced with permission from Science [4].

the backbones of polyisoprene and polybutadiene) and then using a non-selective physical etch (reactive ion etching (RIE) with $\mathrm{CF}_{4}$ ) to transfer the pattern of holes to an underlying semiconductor substrate. A negative mask can be made of the same block copolymer which will generate an array of posts instead of holes. In this case, the polydiene is stained with osmium tetroxide so that the matrix will etch in $\mathrm{CF}_{4}$, while the minority block is now etch-resistant. Similarly, poly(styrene-b-methyl methacrylate) is a promising choice for nanolithographic applications since the nanodomains of poly(methyl methacrylate) may be degraded by radiation and then removed with acetic acid [5] to create a positive mask. While these etching techniques lead to a huge density of holes or pits on the 
surface, achieving a suitable aspect ratio has been a problem due to the spherical shape of the nanodomains which form the mask and the relatively fast etch rate of the polymeric mask. Cylindrical nanodomains have greater aspect ratio features, and have been used to produce dynamic access memory stack capacitors [6-8]. In processing these devices, however, Guarini and coworkers showed that the polymer films must be sufficiently thin $(<45 \mathrm{~nm})$ to demonstrate a high degree of pore-size uniformity. The block copolymer spherical domains or short cylinders were used to etch into a secondary dielectric mask and then this harder, etch-resistant layer was used as a mask for patterning the underlying substrate [8].

Lift-off techniques which involve the vacuum evaporation of an inorganic material into the nanometer pores of a developed block copolymer mask are probably the most direct way to transfer patterns to fabricate magnetic [9,10] and nanoelectrode arrays [11]. Alternatively, many of the etching techniques used above can be transferred to underlying metal layers. In these cases, however, the etch contrast between the developed and undeveloped regions must be quite large due to the slower etch rate of the metals. Polyferrocenylsilanes (PFS) have a significantly lower etch rate than most organic polymers, so selective etching of the PS domains in a PS-b-PFS block copolymer happens automatically and yields high aspect ratios [12-15]. Using a multilayer process, this pattern can be transferred first into a dielectric such as $\mathrm{SiO}_{x}$ and then into a magnetic material, such as cobalt, nickel or iron, for use as magnetic data storage media, as shown in Fig. 4 [12,13]. Control of the longrange order of the PFS spherical nanodomains necessary for data storage applications has been demonstrated by Cheng et al. and will be discussed in detail later in this article [16,17]. For similar applications, Russell and coworkers have used RIE to transfer the pattern from a nanoporous PS film into a $\mathrm{FeF}_{2} / \mathrm{Fe}$ bilayer [18], while Asakawa and coworkers have used a spin-on-glass to create an intermediate mask from a similar nanoporous PS film and then used the glass dots as a mask to ion mill an underlying magnetic film $[19,20]$. Ober and coworkers have explored the use of small molecule additives to enhance the phase selective chemistry available in block copolymer systems, merging the block copolymer lithographic routes with more standard light-driven pattern development processes of the microelectronics industry [21].

\subsubsection{Nanostamping and imprinting}

Siloxane-based elastomers have found widespread use as stamps for transferring patterns in optical systems, microelectronics, and sensors. In most cases, these stamps are manufactured by lithographically patterning a mold, filling the channels or pores with a poly(dimethylsiloxane) (PDMS) prepolymer, curing, and then releasing the stamp from the mold for future use. Recently, there have been a few schemes demonstrated for patterning a nanometer-scale stamp using a block copolymer mold. The pores formed by a PMMA-b-PS mask, similar to that discussed in the last section, serve as the features for these molds. Despite optimization of the stamp filling process involving an increase in the hardness of the PS mold via crosslinking, the use of a PDMS prepolymer dissolved in toluene to enhance wetting of the $20 \mathrm{~nm}$ pores, and the use of an electric field driving force to enhance pore filling, posts in the stamp were only $6 \mathrm{~nm}$ high in comparison to the $40 \mathrm{~nm}$ deep pores in the mold. This example reveals a fundamental challenge that must be met by this method, i.e. displacing air to fill $20 \mathrm{~nm}$ features of the mold with a viscous prepolymer. This seems to indicate that any route towards stamp development on this length scale will be plagued by similar issues, regardless of whether the master is made via block copolymer patterning or a more traditional method [22].

Probably, the most viable challenger to block copolymer lithography is nanoimprint lithography, a technique which builds on much older embossing techniques. This method takes advantage of other more expensive or tedious lithographic techniques to pattern a master on a nanometer length scale, but 


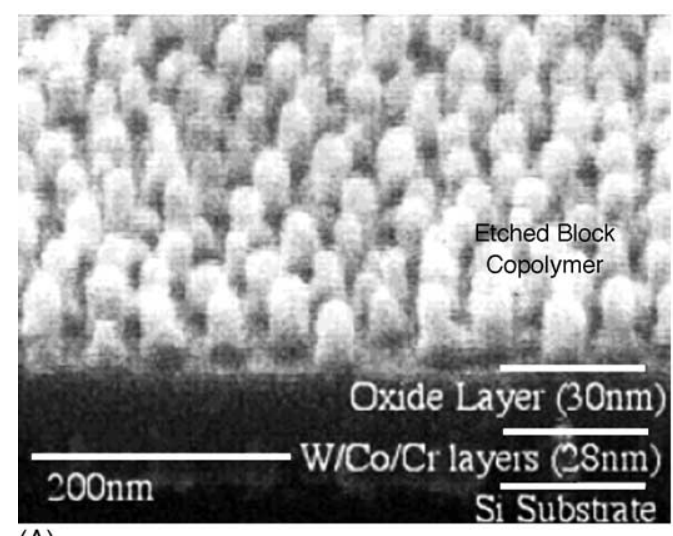

(A)
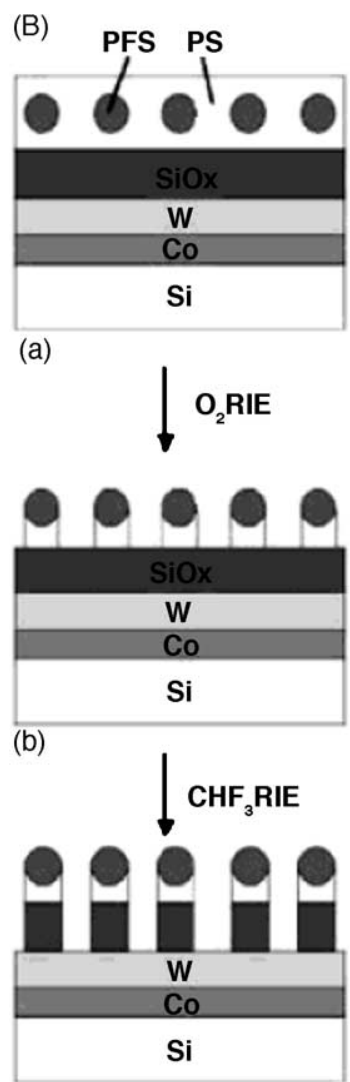

(C)

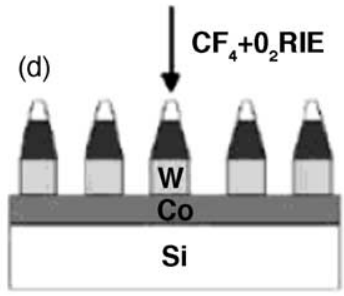

Ashing

(e)

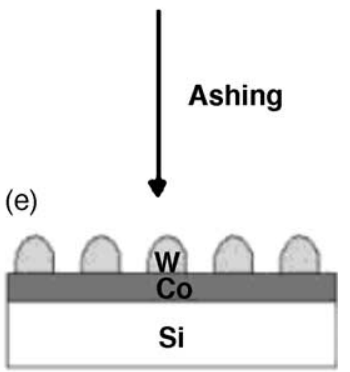

(f)

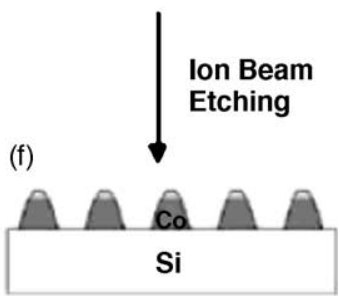

Fig. 4. The inherent etch contrast in a poly(styrene-b-ferrocenylsilane) block copolymer makes it possible to create higher aspect ratio features as shown in the scanning electron micrograph of the mask (A). Schematic (B) demonstrates a route towards magnetic dots applicable to hard drive fabrication using this block copolymer. Reproduced with permission from Advanced Materials [13].

this master may be used repeatedly. In nanoimprint lithography, the master is made out of a hard material such as silicon or nickel. The pattern is transferred from the master (which must be made out of a suitably hard material) to the substrate by pressing it with a relatively large force into a resist that is heated above its glass transition temperature [23]. When the resist is of high viscosity, similar filling problems to those listed above may occur. In this case, however, a transparent master may be used to mold a very low viscosity prepolymer that can be photopolymerized, while still encased in the mold $[24,25]$. Upon separation from the mold, a pattern is left on the surface and can be transferred into an underlying substrate using standard microelectronic processing techniques.

\subsection{Bottom-up techniques}

Recent interest in this field stems from the recognition that block copolymers may enable a wide variety of new applications beyond the traditional semiconductor industry. For applications such as nanofilters and catalysis, a functional material is contained within a block copolymer nanodomain rather than the etching of an underlying substrate as previously discussed. These domains can be obtained by seeding one of the blocks or fully formed nanodomains with the new material or by synthesizing and processing functional copolymers. 


\subsubsection{Incorporation of nanocrystals and nanoreactors}

Quantum size effects and large surface to volume ratios can contribute to the unusual properties of nanoconfined inorganics and studies of these effects have sparked great interest in the fabrication of nanometer-scale particles of metal or metal oxides. The synthesis of nanoparticles from block copolymer nanodomains is an effective route to controlling the size distributions, shapes, and placements of the nanoparticles. The synthesis may be performed by binding the inorganic (either physically or chemically) to the polymer backbone prior to self-assembly or by loading an already formed nanodomain. In the simplest renditions, one may choose blocks with higher affinities to attract the inorganic. For instance, Jaeger and coworkers demonstrate that metals deposited directly to the surface of a film would segregate into the underlying nanodomains. Hydrophilic blocks such as PEO can absorb reactants such as $\mathrm{SiCl}_{4}$ which can be used to grow $\mathrm{SiO}_{2}$ posts directly within the domain, removing the development step within the lithographic process [26-28].

More intricate mechanisms make use of metal-containing polymers which are directly selfassembled. Cohen and coworkers attached metals to the backbones of norbornene-based polymers which then self-assembled into the expected spheres, cylinders and lamellae when cast into thin films and heated to allow equilibration. These inorganic nanodots serve as reactors and can be converted to a variety of metal oxides, chalogenides, or zero-valent metals [29-33]. This method was later generalized to self-assemble a carboxyl functionalized norbornene polymer into which the metal salts could later be loaded by soaking for a period of several weeks. Poly(2- or 4-vinyl pyridine) (PVP) block copolymers are particularly able to complex with many metal salts due to the presence of the nitrogen in either the two or four position of the aromatic ring. Solution phase routes for fabricating palladium [34], cobalt [35], gold [36], rhodium [37], and platinum nanoclusters [37] in the P4VP core of a PS-bP4VP block copolymer micelle by the reduction of a metal $\left(\mathrm{CH}_{3} \mathrm{COO}\right)_{2}$ have been investigated for catalysis applications. Using a similar method which eliminates high-temperature heating steps, Ahmed et al. have made very regular $\mathrm{CoFe}_{2} \mathrm{O}_{4}$ nanoparticles which are ferrimagnetic at room temperature [38]. The introduction of inorganic precursors can be accomplished directly from a preorganized block copolymer film by exposing the film to a metal-containing gas [39] often yielding ordered arrays more appropriate for nanotemplating applications. Brown and Watkins have used supercritical $\mathrm{CO}_{2}$ to transport platinum and silver complexes to the P2VP phase of a PS-b-P2VP block copolymer where it was subsequently reduced [40]. Sohn and Seo sequestered gold nanoparticles into the P4VP lamellae of a PS-P4VP diblock copolymer thin film by making use of the strong interfacial segregation of the blocks [41]. Horiuchi et al. fabricated palladium or cobalt nanoparticles in preformed films of a variety of block copolymers by exposing the films to vapors of $\mathrm{Pd}\left(\mathrm{CH}_{3} \mathrm{COCH}-\right.$ $\left.\mathrm{COCH}_{3}\right)_{2}$ or $\mathrm{Co}\left(\mathrm{CH}_{3} \mathrm{COCHCOCH}_{3}\right)_{2}$ and then reducing these metalorganic species. The nanoparticle always formed in the block copolymer nanodomain with the stronger reducing power [42]. Manners and coworkers have demonstrated the synthesis of ceramic nanolines based on PS-PFS block copolymers $[43,44]$. More recently, this group has also shown that ferromagnetic ceramic films can be formed directly by the plasma treatment of a metallopolymer (a cobalt modified PFS block copolymer) [45].

Block copolymers in solution have also been used to form metal laden micelles which may be ordered on surfaces. Spatz et al. [46] generated quasiregular arrays of nanometer-sized noble metal and metal oxide clusters on flat substrates first forming micelles with polar cores from polystyreneblock-poly(2-vinyl pyridine) in toluene. The micelle cores could then be loaded with a metal precursor and reduced to form elemental or oxidic particles. A monolayer of hexagonally arranged micelles was transferred to a substrate by dipping the substrate into a dilute solution. The size (height) of the clusters could be varied between 1 and $15 \mathrm{~nm}$ depending on the concentration of the metal salt. The interparticle distance could be varied between 30 and $140 \mathrm{~nm}$ by using block copolymers with 
different lengths of the blocks. Nanoparticles fabricated via similar routes have been used as catalysts [47], lithographic masks [48]. For instance, this approach has been extended to create diamond nanocolumns for use as cold cathode emitters [49]. Lee et al. deposited similarly fabricated cobalt nanoparticles onto a substrate pre-patterned with a periodic array of holes. The pattern geometry generated capillary forces which selectively positioned the nanoparticles within the holes. As a result, a controllable number of particles could be placed per lattice point [50]. In a similar combination of bottom-up and top-down approaches, Sidorenko et al. used the supramolecular assembly of $4^{\prime}$ hydroxybenzeneazo benzoic acid (HABA) with a poly(styrene-b-4-vinyl pyridine) block copolymer, to form a nanochannel network lined with reactive P4VP. The channels were filled with Ni clusters via electrodeposition to fabricate the ordered array of metallic nanodots [51].

\subsubsection{Ordering of functional block copolymers}

The original self-consistent mean field simulations assumed symmetry in statistical segment length between the blocks but this not necessarily a valid assumption for many functional systems. The molecular basis for constructing free energy expressions that account for the subtle variations in mixing entropy and enthalpy that influence miscibility has been the subject of lively debate. In the case of two flexible polyolefins, a contrast in statistical segment length had a profound impact on phase behavior [52]. Bates and coworkers propose that the conformational asymmetry contribution to $\chi$ is purely entropic [53-55]. For a large set of polymer coils, the phase behavior can instead be rationalized through a self-consistent set of solubility parameters (which vary linearly with statistical segment length) [56-60]. The non-ideal behavior of more than a dozen model polyolefin mixtures have been ascribed to this difference in pure component solubility parameters, but the model does ignore changes in inter- or intrachain structures that may occur due to variations in local crowding that may account for the mixtures that do not fit the model. These molecular pairs may be influenced by an enthalpic contribution to $\chi$ through the effective coordination number which is maximized when both blocks are elongated [60]. Fascinating self-assembled structures observed by Chen et al. indicate that the presence of a much stiffer rod block fundamentally affects microphase separation [61]. Many simulations predict that as the stiffness of one of the blocks is significantly increased, a new class of intermolecular interactions is introduced, in particular the display of liquid crystalline phases such as nematic or layered smectic structures with molecules arranged with their long axes nearly parallel to each other [62-69]. While the complete phase diagram of these types of molecules is not yet known, the chain rigidity of the stiff block is expected to greatly affect the details of the molecular packing and thus the nature of thermodynamically stable microphases. The high stiffness contrast between the blocks has been observed to drive microphase separation even at very low molecular weights as compared to flexible block copolymers. The supramolecular structures of rod-coil polymers arise from a combination of organizing forces including the repulsion of the dissimilar blocks and the packing constraints imposed by the connectivity of the blocks, and the tendency of the rod block to become orientationally ordered. It is not surprising that many unusual symmetries such as zigzag and arrowhead phases have been observed [61,70]. The thin film structure, however, of rod-coil block copolymers is not nearly as well understood or reproducible as that of the coil-coil type blocks discussed elsewhere in this review.

Self-assembling biological block copolymers, such as block copolypeptides and phospholipids can be prepared from renewable resources, are biocompatible and biodegradable, and are good examples of self-assembly with several added degrees of complexity when compared to traditional block copolymers. Peptide folding, for instance, is not only controlled by the sequence of monomer units, but also by specific monomer-monomer interactions (secondary or $\beta$-structure). Materials with properties that more closely approach the complexity found in biological systems are currently being 
synthesized and processed into ordered assemblies for applications in tissue engineering, drug delivery, and templating of more intricate structures [71,72]. Many of the current applications of these biomimetic block copolymers are in an aqueous solution phase [73-75]. Amphiphilic block copolypeptides have also been shown to self-assemble into dilute but rigid hydrogels that exhibit nanoscale and microscale porosity (important for potential tissue engineering applications). These also exhibit shear thinning rheological behavior and rapid recovery after the cessation of shear and can be built with specific biofunctionality [76]. Potentially, solid-state self-assembly, similar to that used in traditional block copolymers, could be used to form ordered materials useful as fibers, elastomers or stimuli responsive materials [77-79]. A broad discussion of the self-assembly and potential applications of this important class of block copolymers is beyond the scope of this review, though several other interesting reviews focused on this subject have been recently published $[71,72,75]$.

Conductive polymers with $\pi$-bonded backbones are another example of a non-coil-shaped polymer system in which block copolymer templating may be applied to great advantage in applications. Recent breakthroughs in the device physics of these materials have demonstrated that the interfaces between two conducting organics of different work function play an integral role in the separation of electrons and holes to generate energy in a photovoltaic cell or conversely in the recombination necessary to generate light. Friend and coworkers demonstrated that blending of conducting organics led to a four-fold increase in the efficiency of LEDs [80]. Heeger and coworkers have also demonstrated that blending a conjugated polymer with a water-soluble ion-transporting polymer, poly(ethylene oxide), produces efficient luminescent electrochemical cells [81]. Hadziioannou and coworkers have demonstrated the formation of photovoltaic cells made from electron donating/electron accepting block copolymers [82-84]. Essential to all of these devices is controllable, predictable pattern formation which is based on phase separation. Several groups have observed the self-assembly of block copolymers formed from a conjugated rod block covalently bonded to nonconjugated coil block. Currently, these materials have been reported to self-assemble into a large number of novel structures. Yu and coworkers reported lamellar organization of spun-cast thin films of a rod-coil block copolymer containing oligo(phenylene vinylene) (PPV) derivatives as the rod blocks and polyisoprene or poly(ethylene oxide) as the coil block [85]. Other conjugated rod-coil block copolymers have been seen to assemble into ribbon-like fibrils [86] and non-spherical micelles [87]. Rod-coil triblocks containing oligo(phenylene vinylene) synthesized by Stupp and coworkers have been observed to self-assemble into mushroom shapes in solution [88-90], while poly(phenylquinoline-b-styrene) copolymers form hollow spheres when cast from specific solvent mixtures [91]. One of the main challenges to understanding and controlling order in these highly segregated, complex systems lies in obtaining and distinguishing equilibrium. Many of the above systems were solvent-cast and trapped in non-equilibrium structures as the solvent evaporated. The same may be true for thermotropic systems in which a rapid quench in temperature across liquid crystalline phase transitions can result in trapping. In light of the extraordinary progress made towards controlling order in classic block copolymers, it is entirely expected that in the near future the phase diagram of these unusual rod-coil block copolymers will become clearer and evidence of successfully templated and controlled structures of use for optoelectronics will appear.

Block copolymers are attractive electrolytes for solid-state batteries in which the ionic conductivity of a liquid, the mechanical properties of a solid, and the formability of a commodity thermoplastic are desired. Additionally, the polymer (or block therein) must have a high dielectric constant and chain mobility at room temperature. By choosing a lithium salt-solvating polymer as one block component, continuous ion-conducting pathways can be templated. Poly(ethylene oxide) is a popular choice for the ionically conductive block when combined with a stiff, high-Tg block for dimensional stability [92]. Many alternatives for the PEO block have been suggested and are discussed 
in a recent review [93]. The microphase separated, ordered morphology may also be used to lend solidlike mechanical properties even above the glass transition temperatures of both blocks. Soo et al. have demonstrated that a self-assembled poly(oligooxyethylene methacrylate)-b-poly(laurel methacrylate) (POEM-b-PLMA) block copolymer is mechanically stable and has an ionic conductivity similar to the molten homopolymer POEM [94]. The presence of salts in the polyelectrolytes for battery and fuel cell applications, however, affects both the conformation of the chain and the $\chi$ parameter of any block copolymer with a polyelectrolyte block $[95,96]$. The thermodynamic self-assembly of a poly(ethylene oxide)-based block copolymer can also provide a template for incorporation of gold salts or nanotubes to create a self-organizing, nanocomposite electrode [97]. Bullock and Kofinas have used ionically conducting block copolymers as templates for the synthesis of lithium manganese oxide nanoparticles such that a self-assembled film functions as both a polyelectrolyte and as a composite anode in a lithium battery system $[98,99]$.

Directly self-assembling functional structures may simplify fabrication processes and allow for new applications. Most of our knowledge of block copolymer self-assembly, however, relies on classic organic backbone polymers. The special properties of inorganic backbones provide new handles to be tuned for order control. Eitouni et al. have demonstrated that the $\chi$ of a poly(styrene-b-ferrocenyldimethylsilane) polymer is similar to that of poly(styrene-b-isoprene) and when the redox states of $8 \%$ of the ferrocene moieties are changed, the order-disorder transition temperature can be reduced by as much as $40{ }^{\circ} \mathrm{C}$ [100]. These stimuli responsive materials may be very useful in applications requiring multiple switches, such as actuators and photonics. Cheng et al. have demonstrated that block copolymer templating techniques may be used on these types of systems, although they appear to order only over a shorter length scale [101]. Hinderling et al. have used a similar PFDMS block copolymer as the catalyst for the growth of carbon nanotubes of controlled width. In this application, the nanotube will have a diameter equal to the radius of the spherical catalyst domain, which can in turn be tuned by the polymer chemistry. As a result, they have demonstrated an interesting new route to the controlled growth of diameter tuned nanotubes, as shown in Fig. 5 [102].

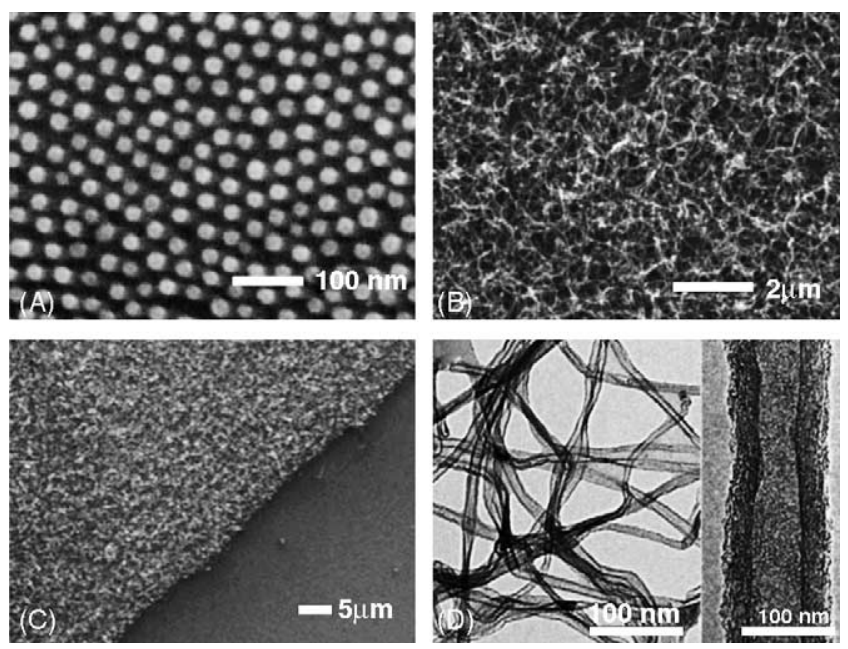

Fig. 5. Block copolymer ferrocenosilane domains act as catalysts for carbon nanotube growth allowing for easy tuning of nanotube diameter via alterations in molecular weight of the block copolymer. (A) SEM image of the iron-containing nanodomains on a silicon substrate after reactive ion etching removal of the organic matrix. (B) SEM image of a surface after growth of carbon nanotubes. Part (C) demonstrates that when the polymeric film is scratched from an area, no nanotubes are templated. (D) TEM images of two different resolutions of nanotube growth. Reproduced with permission from Advanced Materials [102]. 


\section{Imaging and analyzing nanometer-scale order}

Classic studies concerning the organization of block copolymers utilize small angle scattering (X-ray or neutron) to analyze long-range three-dimensional order and transmission electron microscopy (TEM) to observe real-space pattern formation. The addition of a hard substrate in the thin film morphology creates a difference between the in-plane and out-of-plane directions in the organization of the thin film. The direction perpendicular to the substrate is well studied by a variety of depth profiling techniques including secondary ion mass spectroscopy, time-of-flight forward recoil spectrometry, nuclear reaction analysis, and X-ray or neutron reflectivity. A comprehensive review of both real-space and momentum space profiling is covered in greater depth in a previous review [103].

The detailed structure including grain defects such as disclinations and dislocations are also important to applications involving lithographic templating. Techniques from crystallography are finding their place in block copolymer order analysis. Transmission electron microscopy of the inplane structure of a film may be performed by delaminating the film from its non-transparent substrate and "floating" onto a dummy substrate such as a copper grid. Morkved et al. used silicon nitride windows to create solid substrates on which block copolymer layers could be imaged through TEM [104]. Both scanning electron microscopy and atomic force microscopy can be used to visualize the real-space locations of nanodomains if they can be resolved from the top of the film. Scanning probe microscopies easily image contrasts in moduli of the top layer of a film and may sometimes be aided by selective swelling of one of the materials to create further contrast. Both SEM and SPM techniques, however, only image the top of a film. Reactive ion etching can be used to view materials that have glassy surface layers or to etch to the midplane of the layer of interest. Correlation of images from multiple layers of nanodomains can yield information on the three-dimensional structure of a thin film $[105,106]$. Mathematical treatments of images of the nanodomains can quantify the degrees of order observed. Voronoi diagrams and Delauney triangulations can be used to count the number of nearest neighbors of spheres or cylinders in crystalline lattice and simplify the process of pinpointing dislocations and grain boundaries [90,107,108]. Using these tools, Hammond et al. statistically illustrated that cylinders in a dislocation core modify their radii to suit the pressure field around them [109]. Correlation functions quantify degrees of translational and orientational order within any single crystal allowing comparisons of order [110,111]. Caution must be exercised in each of these calculations as small hystereses, such as piezo creep in SPM imaging which could be ignored in qualitative analyses of order, are major effects in quantitative imaging. A variety of methods have been introduced to correct images for these small errors to minimize this problem [112,113].

In well-organized samples, the grain size may be larger than 50 times the domain spacing, and images which are large enough to encompass several grains and allow a measurement of grain size do not resolve individual nanodomains. In some cases, the "sewing" together of several images may be used to illustrate grain size, but this effect is slow, tedious, and prone to inaccuracy. Angelescu et al. have used undersampled microscopy to analyze grain boundaries in two dimensions [114]. The overlaying of a $512 \times 512$ pixel imaging array on a randomly oriented hexagonal grain creates a Moiré pattern as shown in Fig. 6. Hexemer et al. used an AFM with minimized thermal drift to create Moiré patterns in which it was possible to locate dislocations and other point defects in two-dimensional block copolymer arrays [113].

Most of the in-plane analysis techniques described above are only capable of observing the organization of the upper-most surface (sometimes following an etch). Order within the plane of the film is an essential component in nanopatterning applications. Grazing incidence small angle X-ray or neutron scattering (GISAXS or GISANS, respectively) provides information on the lateral and internal ordering of a thin film averaged over the length of the sample. Unlike standard small angle scattering 


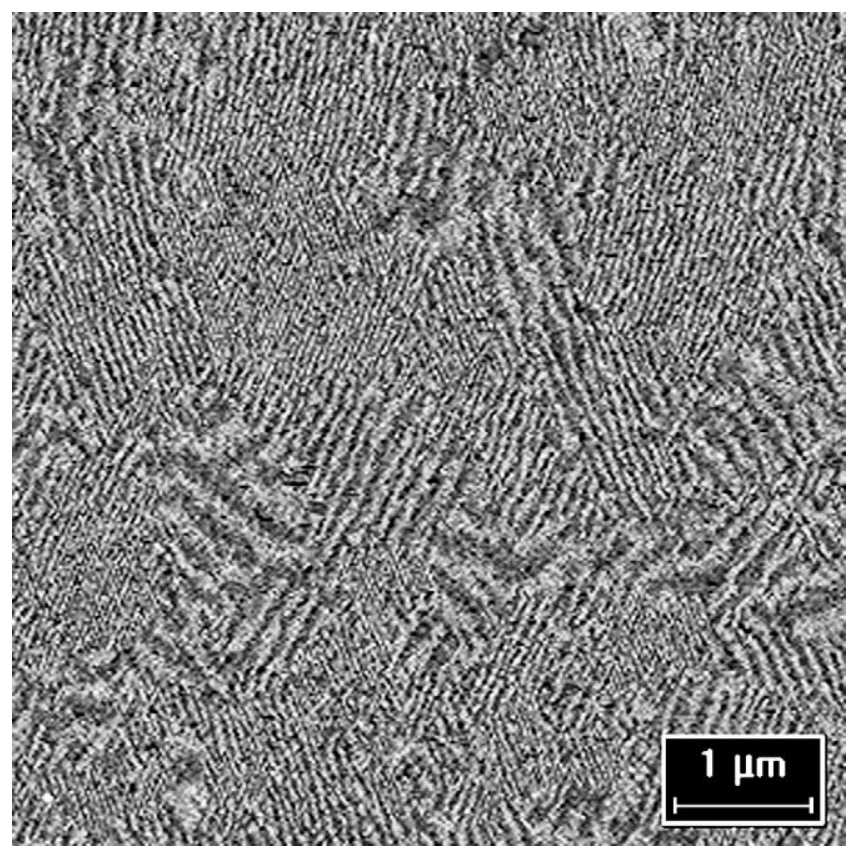

Fig. 6. Moiré pattern of a sphere-forming block copolymer. The undersampled image provides information on grain sizes and shapes resolving individual nanodomains. Reproduced with permission from Applied Physics A: Materials Science \& Processing [114].

techniques, this technique is not hindered by the presence of a non-X-ray transparent substrate since the data are acquired in a reflection geometry. The result is a Fourier space picture of the long-range order of the materials which includes information on buried structures frequently unobservable with AFM [115,116]. This technique is also useful for observing the real time evolution of order in polymer films [90].

\section{Film casting}

The first obstacle to studying and reproducing order within a self-assembling thin film is casting a film which is of constant thickness and smooth over a large area. Below are outlined a few of the common casting techniques and a discussion of how thermal equilibrium in the films can be achieved.

\subsection{Inducing order during film formation}

Essential to most polymer thin film applications is the long-range order of the nanoscale features. In principle, the self-assembly process leads to regular, stable organization because the structure is close to thermodynamic equilibrium. Both dip coating and solution casting are slow processes which allow self-assembly to occur during the casting process. Furthermore, the non-equilibrium effects of solvent evaporation actually lend an orientational field leading to interesting morphologies. For instance, the orientation of block copolymer cylinders changes with the evaporation rate of the solvent [117]. More commonly, however, block copolymer thin films are prepared by spin-coating. In this process, a drop of dilute polymer solution ( $\sim 1 \%$ by weight $)$ is dropped on a spinning solid substrate. As the substrate spins, all but a boundary layer of the solution is spun off. The thickness of the 
boundary layer depends on concentration and spin speed and these variables therefore control the thickness of the final film. The surface roughness of both spun-cast and solvent-cast films can be controlled by varying the evaporation rate of the solvent. When the solvent is too volatile, the surface is roughened by flow instabilities [118]. As will be discussed later, the physical behavior of the solvent can also influence the order of the block copolymer. In particular, when the solvent is crystallizable, directional crystallization of the solvent can influence the orientation of the block copolymer selfassembly. Since the solvent is frequently chosen to maximize film uniformity, the use of truly nonselective solvents is rare. Furthermore, the solvent flashes off so quickly that equilibrium structures are not usually observed. Self-assembly is frequently allowed to occur during a long anneal at elevated temperatures under vacuum. This technique not only removes solvents and minimizes surface-induced non-equilibrium effects, but also increases the chances of a polymer reaching its thermodynamic equilibrium morphology.

As the molecular weights of the block copolymers rise and the architectures become more complex, kinetic barriers to thermodynamic equilibrium become larger obstacles. The trapping of metastable states has been observed numerous times in rod-coil block copolymers. For applications requiring larger domain spacings, extremely large molecular weights are necessary. In strongly segregated melts, the characteristic microdomain spacing $d$ increases approximately as the two-thirds power of molecular weight [119]. Unfortunately, the diffusion coefficient scales as $\exp (-\alpha \chi N)$ which means that the rearrangement process quickly become glacially slow as the domain length increases [242]. Yokoyama et al., for instance, demonstrate that the time required for a single chain to hop from one set of block copolymer spheres to another was on the order of minutes [148]. As a result, the temperature window between glass transition and decomposition is often insufficient for thermal equilibration. In these situations, several groups have proposed solvent vapor annealing of thin films $[117,120-122]$. The vapor acts as a plasticizer and provides mobility to the film without a significant increase in temperature. The solvent can also screen unfavorable interactions between the segments leading to a depression in the order-disorder transition [123,124]. Since completely non-selective organic solvents are rare and selectivity leads to an apparent change in the composition of the block copolymer, it is not surprising that solvent annealed films are organized differently than their thermally annealed counterparts. Furthermore, the evaporation of the solvent is highly directional, and can be used to induce orientation. For instance, flow normal to the pinned contact line, leads to well-ordered, highly aligned arrays of cylindrical nanodomains over lateral length scales of several tens of micrometers [125]. Krausch and coworkers demonstrated both that the choice of solvents dramatically impacts the kinds of structures observed and also that the rate of solvent removal plays a role in the orientation of the nanodomains [126,127]. Cylinder-forming diblock copolymers demonstrate better order when vapor annealed, but are more sensitive to aging effects than thermally annealed counterparts [128]. Although compressed fluids are generally poor solvents, supercritical $\mathrm{CO}_{2}$ has recently been demonstrated as an efficient plasticizer for block copolymer annealing [129-131]. The absorption of the compressed gas by the film may be controlled precisely through the fluid density at very low volume fractions. The presence of $\mathrm{CO}_{2}$ has been observed to have a surprising effect on the miscibility of two blocks, but little is known about the long-range ordering of thin film structures during this type of anneal $[130,131]$.

\subsection{Film thickness}

A difference in block interfacial energies will attract one of the blocks to the substrate, inducing a layering effect on the remainder of the film [132-137]. Lamellar films possess a natural repeat spacing of the domain structure, $L_{0}$, and pay a free energy penalty due to chain stretching or compression when 

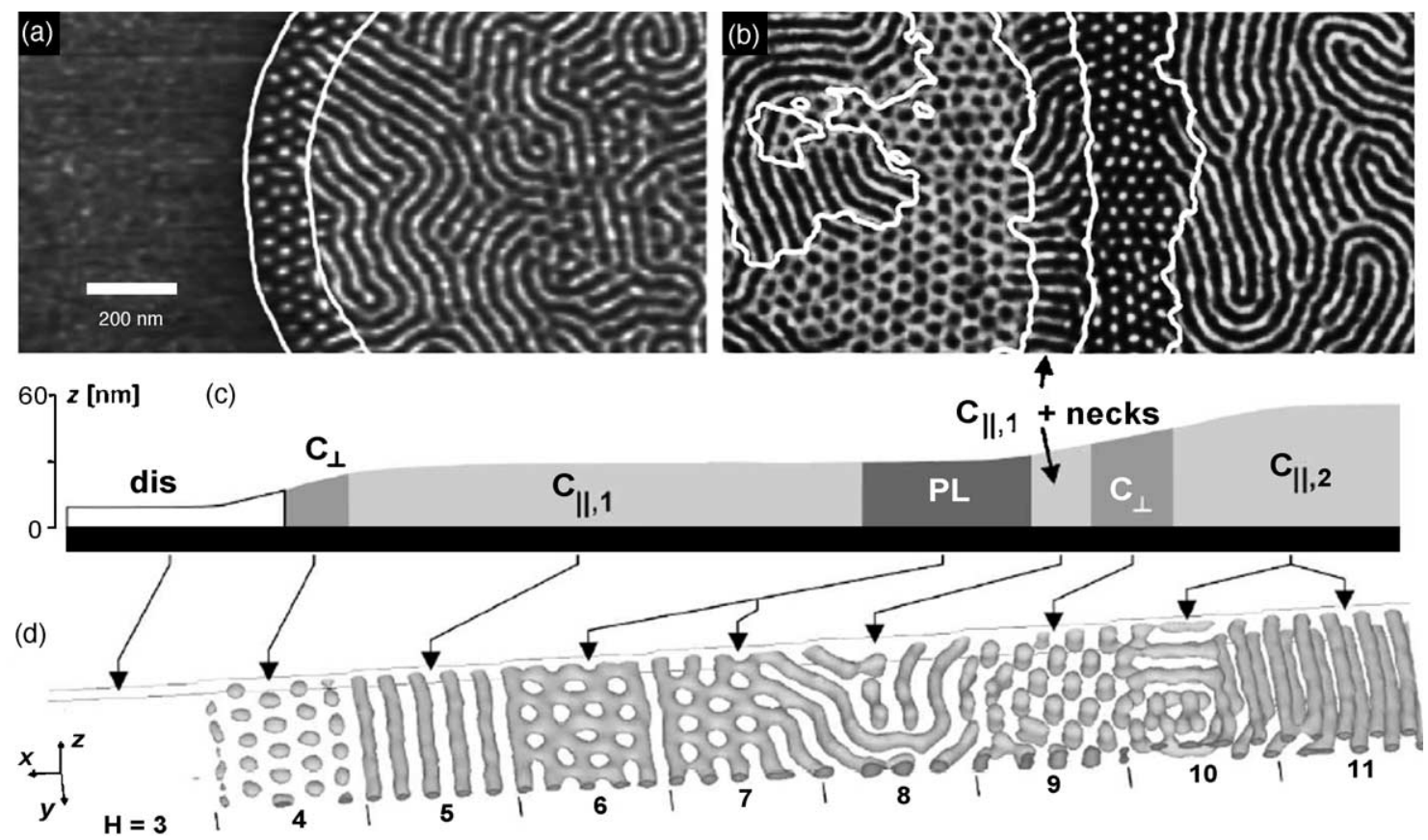

Fig. 7. The self-assembly of block copolymers is fundamentally influenced by the boundary conditions including film thickness. Parts (a) and (b) are phase SPM images of a thin poly(styrene-b-butadiene-b-styrene) triblock copolymer which reconstructs as a function of film thickness. Part (c) is a schematic height profile of the phase images shown above, while part (d) is a simulation of the same block copolymer. Reproduced with permission from Physical Review Letters [138].

the film thickness is not commensurate with this spacing. This results in the generation of islands and holes (surface structures) at the polymer/air interface. Smooth films are observed after annealing above the glass transition only when the original, spun-cast film thickness matches a natural thickness $(h)$. When both surfaces attract the same block, $h$ is equal to $n L_{0}$, where $n$ is an integer. When the two interfaces attract different blocks, flat films only occur when the initial film thickness equals $(n+1 /$ 2) $L_{0}$. In sphere- and cylinder-forming block copolymers, islands and holes form when the film thickness does not closely match a natural thickness given approximately by $h=\alpha n+\beta$, where $\alpha$ is the sphere (or cylinder) layer-to-layer distance and $\beta$ is the thickness of the brush adsorbed on the surfaces (if such a brush forms).

In very thin films, these surface effects can cause significant deviations from the predicted bulk structure. As shown in Fig. 7, surface reconstructions of a cylindrical block copolymer including reorientation of nanodomains, wetting layers, and perforated lamellae have been observed due to the interplay between surface fields and confinement effects [138].

Recently, surface patterning has been employed by several groups to control island structures. For sub-lamellar thickness films, corrugations in the substrate with repeat spacings on the lamellar size scale create thickness modulations in the overlying polymer film and have been shown to have dramatic effects on lamellar orientation $[139,140]$. Heier et al. have succeeded in templating surface domain structures on smooth substrates that were chemically patterned by microcontact printing on a scale of 1-16 $\mu \mathrm{m}$ [141]. Similar patterning of island and holes has been shown for sphere-forming [142] and lamellar [143] block copolymers on a topographically patterned substrates. Several more studies have investigated the effect of surface patterning on block copolymer orientation and order and these will be discussed later in this review. 


\subsection{Evolution of order}

For all the applications listed above, block copolymers are being utilized in part for their ability to form regularly sized and regularly spaced nanodomains. Even though a large number of equilibrium morphologies are expected, it may take a very long time to reach the equilibrium structure. During this process, one may track several events: (1) the formation of equilibrium nanodomains; (2) the arrangement of these nanodomains into crystalline arrays of increasing size (grain boundary annihilation); and (3) the removal of defects such as disclinations and dislocations.

\subsubsection{Thicker films and bulk order evolution}

Ordering kinetics have been examined in bulk samples but a mechanistic understanding of coarsening dynamics has failed to emerge due to the complexities of defect motions that cannot be easily discerned in bulk samples. Coarsening kinetics in polystyrene-polyisoprene bulk systems have been studied by Balsara and coworkers in bulk systems via depolarized light scattering. They found a slowing of grain growth with time and suggested that grain boundaries gradually became pinned [144,145]. It is necessary, however, to balance the kinetics of pattern formation with the thermodynamics of block copolymer segregation in order to achieve even higher degrees of order. For instance, Kim et al. demonstrated that grain growth rates can be optimized by correctly tuning the quench depth of a bulk sample from the order-disorder temperature due again to an interplay between thermodynamic driving forces and viscous resistance [146]. Recently, Hashimoto et al. have shown that a single grain lamellar microdomain orientation can be achieved using an applied temperature gradient. Also in this case, the process is very slow ( $2 \mathrm{~mm} /$ day) [147]. Yokoyama et al. showed that in the three-dimensional case of very thick films of block copolymer spheres, the interplay between kinetics and thermodynamics controls the extent to which layers of spheres stack with registry to the surface [148]. The degree to which the two blocks will segregate thermodynamically is proportional to $\chi N$. The lower the temperature, the stronger the driving force causing the two blocks to segregate and order. Diffusion of chains depends strongly on the geometry of the nanodomains. In the case of lamellar, cylindrical, and gyroid domains, diffusion along an interface occurs by a different mechanism than that perpendicular to the interface. In the perpendicular case as well as in diffusion between spherical nanodomains, the diffusion coefficient is $D \sim D_{0} \exp \left(\alpha \chi N_{\min }\right)$, where $N_{\min }$ is the shorter of the two blocks [149-152]. At lower temperatures, the polymer may not diffuse fast enough to allow rearrangement of domains into a structure with long-range order. The interplay between these opposite dependences on $\chi N$ results in an optimum annealing condition for thick films of sphereforming block copolymers [148]. This concept should extend to other block copolymer geometries, though this has not been tested.

In the special case of very thin films, local defects may significantly affect the reorganization process. The Ostwald ripening of islands and holes may be easily followed and has provided significant insight into the motion of block copolymers into and out of these structures. Initially, islands and holes form randomly over the sample surface by a process analogous to spinodal decomposition [153,154]. Typically, the average size of the islands increases through coalescence of smaller domains and the growth of larger domains at the expense of smaller ones through an Ostwald ripening process [155-162]. This coarsening is driven by the line tension of the edges of the two-dimensional islands so that over time there is a decrease in the total length of island edges. Heier et al. templated the structure of islands of strongly segregated, lamellae-forming block copolymer using an underlying periodic chemical structure on the substrate formed by microcontact printing. By watching the disappearance of isolated islands near an absorbing boundary, they concluded that the primary mechanism of island shrinkage is the viscous flow of chains along a continuous path created 
by a defect in the lamellar structure within the islands [163] as originally proposed by Grim et al. [164]. Islands and holes of a sphere-forming block copolymer formed on a topographically patterned substrate cannot disappear via a similar mechanism since there is no route for viscous flow of chains even in defect structures. If the island near the absorbing boundary exists only on top of a brush layer, the shrinkage appears to be limited by the detachment kinetics of a chain from the island. If the island is on top of another layer of spheres, the shrinkage of the islands is much faster than would be expected if it were controlled by the diffusion of chains through the underlying nanodomain structure. This indicates the insertion of entire spheres from the island into the layer below, probably through a defect in the underlying film [142]. This is in contrast to the bulk case in which diffusion does not appear to occur through the diffusion of entire spherical domains and instead occurs through the hopping diffusion of individual chains [165].

\subsubsection{Two-dimensional ordering}

The two-dimensional nature of a single layer of nanodomains on a surface also simplifies the task of identifying and observing individual defects. The confinement to two dimensions also fundamentally changes the nature of the organization. For instance, in the two-dimensional hexagonal crystal formed from a layer of spheres, dislocations are point defects and exist in equilibrium. As a result, it is not physically possible to have perfect order, even with optimal annealing conditions at long times. Order evolution in two-dimensional systems is historically an area of interest, but true two-dimensional systems can be realized in only a limited set of experimental arrangements such as bubble rafts, magnetic bubbles, and colloids trapped between substrates or on fluid surfaces. A single layer of block copolymer nanodomains on a substrate is a new addition to this class of systems in which ordering occurs on an observable size scale over an accessible length of time within a wide range of temperatures.

Immediately after film formation, thin films exhibit small grains in the case of spherical nanodomains or very short persistence length, in the case of cylinders and lamellae. Each of these arrays demonstrates not only grain boundaries but also a plethora of point and line defects, as shown in both Figs. 8 and 9. Elegant work by Harrison and coworkers on the coarsening dynamics of a cylindrical block copolymer thin film indicates that disclination annihilation generally requires the coherent annihilation of multiple individual disclinations as seen in $[110,166]$. They found that annihilation events required either three or four individual disclinations to collide and the orientational correlation length of the nanodomains grew with the average spacing between disclinations, following a power law $\xi_{2}(t) \sim t^{1 / 4}$ as a function of time $t$. By tracking individual disclinations with time-lapse atomic force microscopy, they found that the dominant mechanisms of disclination annihilation involved tripoles and quadrupoles of disclinations, as seen in Fig. 8. Similarly, after long annealing, the orientational correlation length of a spherical nanodomain lattice exhibits a $t^{1 / 4}$ temporal power law. The dynamics of pattern coarsening appear to involve the interplay of disclinations and lines of dislocations resulting in the collapse of smaller grains that reside on the boundary of two larger grains [108].

In the case of a single layer of block copolymer spheres, dislocations are point defects and exist in equilibrium. Fig. 9 demonstrates a number of the defects observed in two-dimensional hexagonal systems. Dislocations can be created and annihilated pairwise in equilibrium, leading to defectmediated melting similar to that seen in magnetic bubble and colloidal systems [107,167]. At sufficiently high $\chi N_{\min }$, where $N_{\min }$ is the degree of polymerization of the minority block, the kinetic barriers are too great to allow large grain growth and a polycrystalline array with small grain size is seen. At moderate temperatures, large hexagonal grains with a finite number of dislocations were observed. As temperature is raised, the two-dimensional block copolymer crystal melts through the 

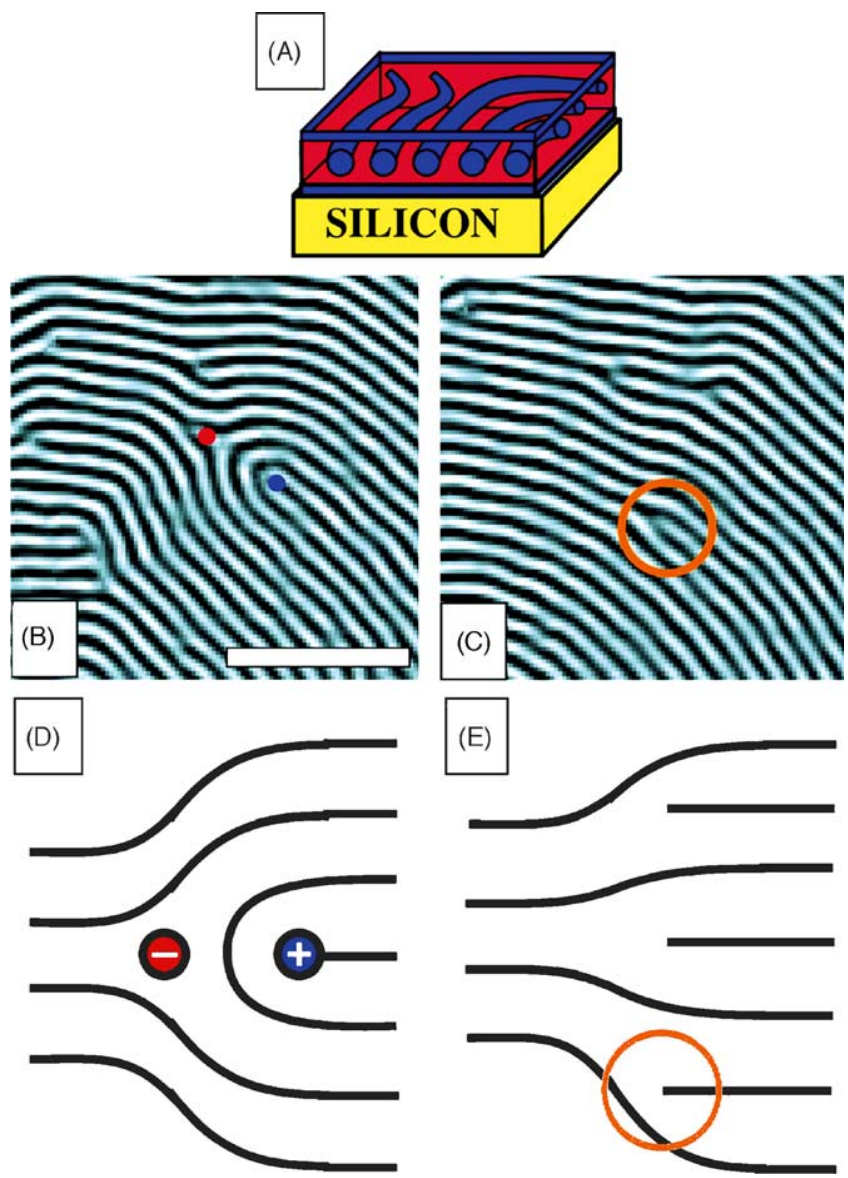

Fig. 8. Defects in a single layer of cylindrical nanodomains lying with their axes parallel to the substrate. (A) Schematic of one layer of polystyrene cylinders in a polyisoprene matrix. (B) AFM image of a disclination dipole consisting of a $+1 / 2$ disclination (blue circle) and a $-1 / 2$ disclination (red circle). Six other dislocations (three each of $+1 / 2$ and $-1 / 2$ ) are visible in the micrograph. The bar indicates $200 \mathrm{~nm}$. (C) After further annealing, the dipole has been annihilated. Four dislocations (one example indicated with the orange circle) of the orientation corresponding to the dipole's original Burgers vector remain, while others have diffused out of the visible range. (D and E) Schematic of disclination dipole annihilation. The strain fields cause the disclinations to annihilate, producing three dislocations (one example indicated with the orange circle) after annihilation in (E). Reproduced with permission from Science [166].

generation and unbinding of dislocation pairs to a hexatic phase followed by the unbinding of the dislocations into their constituent disclinations at higher temperatures resulting in a majority block matrix with randomly arranged spheres [107]. The transitions from a disordered array to a hexatic was observed to be first order [168].

\section{Control of $\mathrm{BC}$ order}

For many of the applications above, it is necessary to have not only a large number of nanometerscale, regularly sized, regularly shaped domains but also to have them organized in a desired fashion. For instance, cylinders aligned with axes perpendicular to a substrate are useful for patterning nanochannels and membranes, while those aligned with their axes parallel to the substrate are useful as lines in nanolithography. Further, if all of the axes are aligned in a single direction rather than 

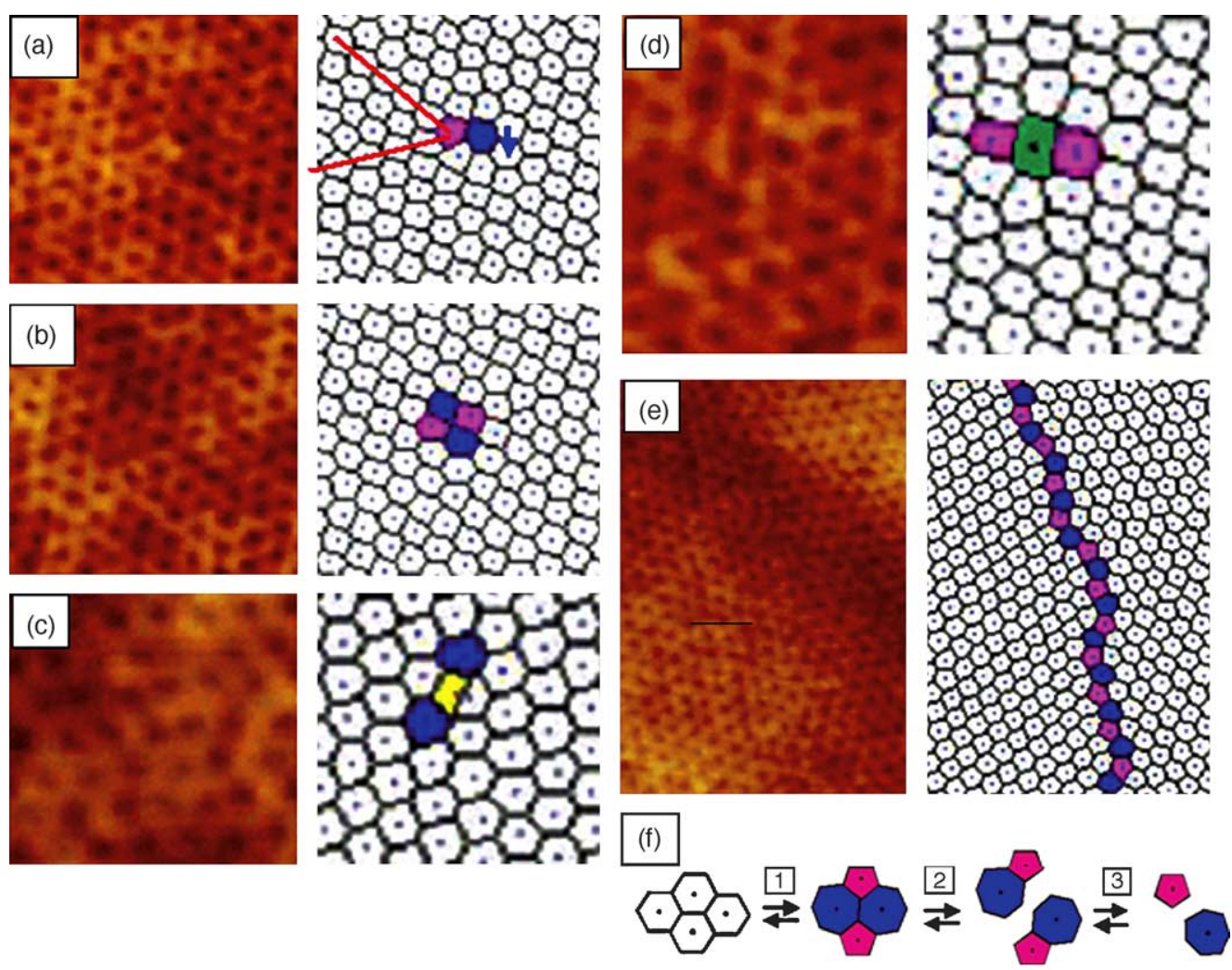

Fig. 9. Defects in hexagonally packed block copolymer spheres. Voronoi diagrams were constructed to count the number of nearest neighbors of any sphere and color coded. White polygons indicate spheres with six nearest neighbors, blues have seven nearest neighbors, magentas have five, yellows have four, and greens have eight. Also shown are corresponding SPM images. (a) Individual dislocation: a paired pentagon and heptagon (5-7 pair) occurs when two extra half rows of spheres (shown as red lines in the Voronoi diagram) intersect at the core of the dislocation. This defect has a Burgers vector in the direction of the indicating arrow. (b) Dislocation pair: in the Voronoi construction, this is seen as a quadrapole of two pentagons and two heptagons (two 5's and two 7's). (c) The 7-4-7 groupings are interstitial spheres (squares are colored yellow). (d) Vacancies are frequently observed as 5-8-5 groupings (octagons are colored green). (e) A grain boundary is seen as a string of dislocations. This is a $30^{\circ}$ grain boundary, corresponding to largest angular mismatch possible and the highest density of 5-7 pairs. (f) A dislocation pair may be formed in equilibrium by the conversion of four hexagons (6's) into a pair of dislocations (two 5's and two 7's) with little mass movement of material and zero net Burgers vector (step 1). These dislocations (5-7 pairs) may then climb and glide away from each other in step 2. Finally, each dislocation may unbind into disclinations (individual 5's and 7's) in step 3. Reproduced with permission from Macromolecules [107].

randomly in the plane parallel to the substrate they may have potential applications in waveguides [169] and dielectric mirrors [170,171]. Spheres arranged in a large hexagonal array may be used to pattern metal dots each of which may be used as an individually addressable bit of memory, but such arrays are only useful if their degree of long-range order is sufficient for reading. The substrates of thin films play a profound role in controlling the orientation of the materials and several methods are summarized below by which either to exploit or to minimize this effect.

\subsection{Epitaxy and surface interactions}

Classically, the crystallographic orientation of a film of a crystallizable material is templated by the substrate. Epitaxy is defined as the growth of a crystal on the surface of another with a orientational relationship between the lattices of the two crystals. This technique has been used 
effectively in controlling the order of block copolymer thin films by modifying the substrate surface.

\subsubsection{Controlling orientation with preferential wetting}

In general, when a block copolymer is deposited on a substrate, one block preferentially wets an interface to minimize interfacial and surface energy. As a result, the lamellae and cylinders are induced to align with their planes (or axes) parallel to the surface due to a preferential interaction of one block with the surface and this orientation tends to propagate throughout the thickness of the film. As discussed previously, the conditions of commensuration are fixed by the natural period of the block copolymer nanodomains. When the thickness of the film is incommensurate with the natural period, relatively thin films (thickness only a few natural spacings) undergo quantization of film thickness to form islands and holes. When one block preferentially wets both the substrate and upper surface, symmetric boundary conditions exist, while asymmetric conditions are in existence when different blocks wet the opposite surfaces. Lamellar patterns parallel to the surface are not particularly useful in many nanopatterning applications, so many efforts center on the reorientation of these types of domains.

The confinement of a non-commensurate film between two surfaces prohibits the formation of islands and holes. The block copolymer chains within the film are either stretched or compressed to compensate for the deviation and the natural domain spacing is perturbed, but if the preferential surface interaction is strong, the parallel orientation persists [172,173]. In cases of severe frustration where a large incompatibility in period and film thickness induces a large strain on the film, the film reorganizes into a heterogeneous structure in which both parallel and perpendicular lamellae are observed [174]. In contrast, when the specific interactions at the confining edges are reduced (a random copolymer with neutral interactions is used to coat the walls) the frustration is accommodated by a reorientation of the diblock copolymer so that the domains run normal to the substrates [175].

When there is no preferential attraction of a block to the surfaces, the domains may align normal to the surface without any film thickness frustration. Mansky et al. first demonstrated that by casting the film on water, this preferential interaction could be removed resulting in cylinders oriented perpendicular from the surface, but this technique proved too unreliable for further use [176]. Alternatively, the surface of the substrate could be more easily tuned chemically to make it neutral to block copolymer interactions. Mansky and coworkers then demonstrated that a PS-PMMA random copolymer with composition tailored to be neutral to wetting by either block could be attached to the substrate surface and would induce lamellar-forming block copolymers to orient perpendicularly [177-180]. Within the plane of the substrate, the persistence length of the lamellae was quite short and the arrangement was anisotropic. Heier et al. demonstrate that a non-preferential wetting layer may be composed of self-assembled thiol monolayers on gold. This route is advantageous in that the monolayer may be deposited only over short areas using a microcontact printing technique. Within these areas, the blocks may be templated to orient perpendicular or parallel to the substrate depending on the local polarity of the self-assembled monolayer [181].

Sivaniah et al. demonstrated that the surface roughness of the substrate can also mask the surfaceinduced ordering in lamellar BCP films [182]. The lamellae must pay an elastic deformation penalty for conforming to a sufficiently irregular substrate and will in some situations rearrange to stack perpendicularly from the surface [183]. Depending on the surface roughness amplitude and periodicity, phase transitions can occur between the two orientations. Since real surfaces are rarely ideally flat, understanding and characterizing surface roughness may be of great importance in controlling orientation of lamellar phases. This orientational control mechanism, however, relies on the relative 
neutrality of the upper surface of the film. In the case studied here (PS-b-PMMA), this was a good assumption, but it usually does not hold true for other block copolymer systems.

\subsubsection{Epitaxy}

While the above strategies focused on controlling the orientation of the long axes of the lamellar and cylindrical geometries, the placement of the domains is particularly important for many lithographic applications. In classical growth of heteroepitaxial crystal films, atoms or molecules organize themselves on a lattice that bears a well-defined relationship to that of an underlying single crystal. In general, the templating crystal must match the natural periodicity of the adsorbed phase within 10-15\% [184]. In graphoepitaxy, by contrast, the surface relief structure of the substrate directs epitaxial growth of an overlying crystalline film [185]. This relief structure can have a length scale much larger than the lattice parameter of the film and the substrate need not be a single crystal or even crystalline [186-188]. Both techniques have been adapted to pattern the organization of block copolymers.

Heteroepitaxy requires that the substrate be patterned in a commensurate fashion with the material to be templated. In small molecules, this is accomplished by lattice matching the template to the new material. Homopolymer chains align with their chain axes parallel to an alkali halide substrate surface so that crystalline lamellae built from these chains stand normal the surface [189]. De Rosa et al. used a similar strategy to control the microdomain orientation of a semicrystalline poly(ethyleneb-ethylene propylene-b-ethylene) block copolymer thin film. The crystallizable block (polyethylene) was epitaxially templated by a crystalline substrate and this block then templated the orientation of the nanodomain structure [190], as shown schematically in Fig. 10.

Strategies for epitaxially organizing amorphous domains of diblock copolymers on substrates have depended on creating substrate topological or chemical patterns that nearly match the domain structure of the block copolymer [139,140,191]. Nealey and coworkers have used extreme ultraviolet (EUV) interferometric lithography to pattern self-assembled monolayers (SAMs) to match the bulk lamellar domain period of a block copolymer (about $60 \mathrm{~nm}$ in this case) [192]. This patterned monolayer provides a surface energy pattern on top of which a symmetric lamella-forming poly(styrene-block-methyl methacrylate) (PS-b-PMMA), can be spin-coated and annealed. The PMMA

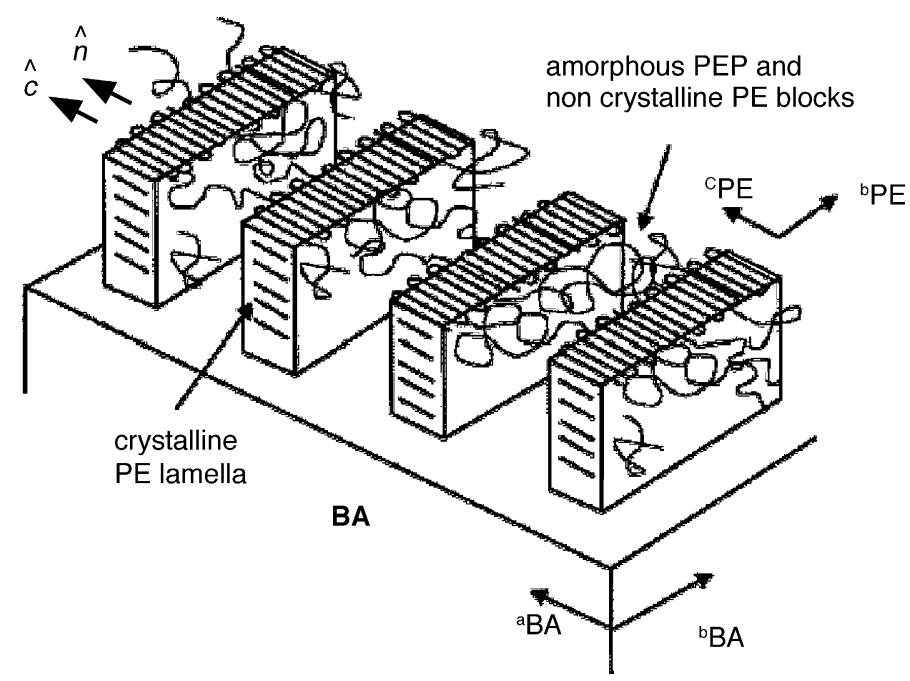

Fig. 10. Schematic model showing the orientation of crystalline and amorphous nanodomains of PE/PEP/PE block copolymer epitaxially crystallized onto benzoic acid (BA). Reproduced with permission from Macromolecules [190]. 


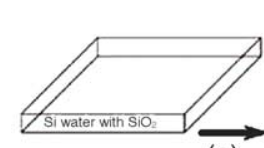

(a)

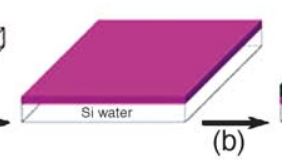

(b)
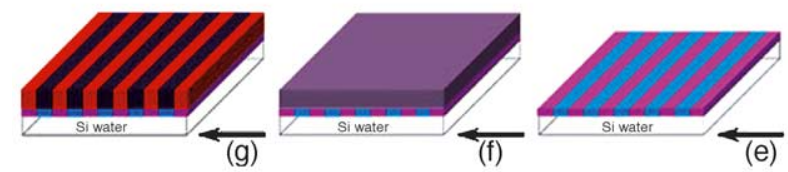

(e)
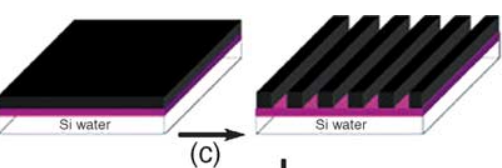

(d)
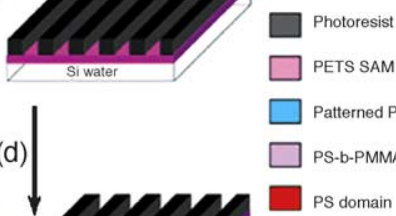

Patterned PETS SAM

PS-b-PMMA

PQ

PMMA domain
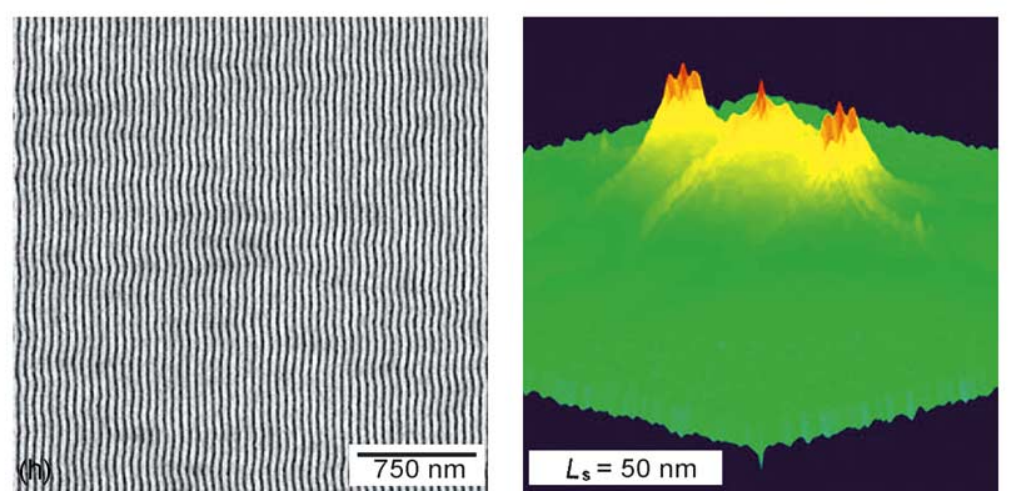

Fig. 11. (a-g) Schematic representation of the nanopatterning of self-assembled monolayers and the subsequent epitaxial assembly of block copolymer nanodomains. (a) A SAM was deposited on a silicon wafer. (b) Photoresist was spin-coated on the SAM-covered substrate and patterned by EUV-interference lithography with alternating lines and spaces of period $L_{\mathrm{s}}$. (d) Irradiation of the sample with soft X-rays in the presence of oxygen transfers the photoresist pattern to the SAM and the photoresist is removed (e). A symmetric, lamella-forming PS-b-PMMA copolymer of period $L_{0}$ is spin-coated onto the patterned SAM surface and annealed (f and g), resulting in surface-directed block copolymer morphologies. If $L_{\mathrm{s}} \sim L_{0}$, the block copolymer will self-assemble to perfectly match the underlying substrate (h). Reproduced with permission from Nature [195].

block preferentially wet the SAMs that were chemically modified to contain polar groups, and the other regions exhibited non-preferential wetting behavior by the block copolymer. When the period of the chemically patterned SAM closely matches that of the block copolymer, the block copolymer orients perfectly on the patterned substrate forming grains several microns in dimension, as shown in Fig. $11[193,194]$. Since the substrate is patterned using a perfect mask and the nanodomains align to match the polymer, it is possible for the dislocation density to be less than the equilibrium value predicted for a two-dimensional crystal on an unpatterned substrate [195]. When the underlying chemical pattern is mismatched in dimension, the block copolymer creates dislocations and other defects to minimize the strain. Simulations suggest that patterning of hexagonally packed cylinders oriented perpendicularly from the substrate should also be possible [196]. Many theoretical predictions of the arrangements of nanodomains on chemically patterned substrates have been made. Chen and Chakrabarti found that commensurability of the natural domain spacing and the period of the pattern is crucial [197]. In cases where this requirement was not met, unequal lamellar spacings [198] and tilted lamellar phases $[199,200]$ are predicted.

Graphoepitaxy represents an advantage over heteroepitaxy in that the surface patterning required is easily accomplished using patterning techniques already in place in the semiconductor industry. As will be discussed, however, the cost is that the order achieved may not be as perfect as that obtained with heteroepitaxial techniques. In the case of block copolymers, graphoepitaxy is achieved by using standard photolithography to pattern a hard substrate and either chemical or physical etching to transfer 
the pattern. This grating serves to induce order away from its edges and has been shown to induce block copolymer spheres to order into a single crystalline matrix over the span of several microns [201]. In the lower portion of the grating (channels), the hard sidewalls template order, while in the raised portions (mesas), the vacuum edge is the nucleating site. At higher temperatures when the spheres are no longer arranged in a crystalline lattice but have lost their translational order to form hexatics and then liquids, the wall serves to increase the orientational order in its vicinity [107,111]. Chaikin and coworkers have shown, through time-lapse studies on a heat-stage AFM that the graphoepitaxial process in the crystalline lattice takes place through the rearrangement of the spheres. Initially, small grains of random orientation form throughout a channel. The preferred orientation (with one close packed row of the hexagonal lattice aligned parallel to the edge) seems to dominate close to the channel edge. Over time, this grain sweeps away from the step edge [202]. Channels with parallel edges form large single crystals since grains nucleated at either side are of the same orientation and can meld seamlessly [201]. In the above cases, the channel width was several orders of magnitude larger than a sphere spacing and commensurability of edge positions with the crystal lattice was not important. Hexemer and coworkers have shown that when the edges do not template the same grain orientation (for instance, the angle between two templating edges is not commensurate with the lattice angles), controlled grain boundaries can be templated [113]. Cheng and coworkers studied the effect of confining spheres into smaller volumes and identified the effect of lithographic defects and the relation of channel width and polymer sphere accommodation [16,17] (Fig. 12). They have found that dislocations and vacancies can be templated by defects in the wall edges or spacings. Wall spacings that do not match an integral number of sphere rows are accommodated by a change in sphere size [243]. Researchers at Toshiba have extended this work for potential application in magnetic data storage using $300 \mathrm{~nm}$ grooves in a spiral pattern which align PMMA spheres [19].

As shown in Fig. 13, Sundrani have recently used graphoepitaxy to guide the assembly of polymer cylinders along the length of the etched channels. Initially, islands and holes form on top of the topographically patterned substrate. In a manner similar to that described earlier in this review for sphere-forming block copolymers [142], material flows from the tops of the mesas to fill the channels. In this case, however, transport may take place via a mass flow along the cylinder axis. As a result, the cylinders are oriented perpendicular to the edges of the channel so that their axes are aligned with the mass flow direction. Further annealing leads to coarsening of alignment across the full trough width at random locations along the troughs, and finally, these smaller perfected regions merge, resulting in perfectly aligned cylindrical domains along the entire channel length. Depending on trough depth and amount of deposited polymer, aligned domains are confined to the channels or extended across the grating with a complete absence of defects [203-205].

Li and Huck have demonstrated the use of nanoimprint lithography for creating the topography for graphoepitaxy directly within a molten polymer layer [206]. Nanoimprint lithography creates features by mechanically deforming the polymer film by pressing a hard mold into the film at temperatures greater than the glass transition temperature of the film. This method is high throughput and inexpensive to apply directly to a spin-coated film of either standing or parallel cylinders. The difficulty in this technique appears to be in keeping the channel walls parallel throughout the imprinted regions. When used on standing cylinders, the patterned arrays show defects similar to those observed by Cheng et al. $[16,17]$ when the width of the channels are not properly tuned and controlled. Similar results were seen when nanoimprint lithography was demonstrated on block copolymer spheres [207].

\subsubsection{Directional crystallization}

As discussed previously in this article, a small molecule solvent in a block copolymer acts as a plasticizer and depresses the order-disorder temperature. Similarly, if both the polymer and solvent 

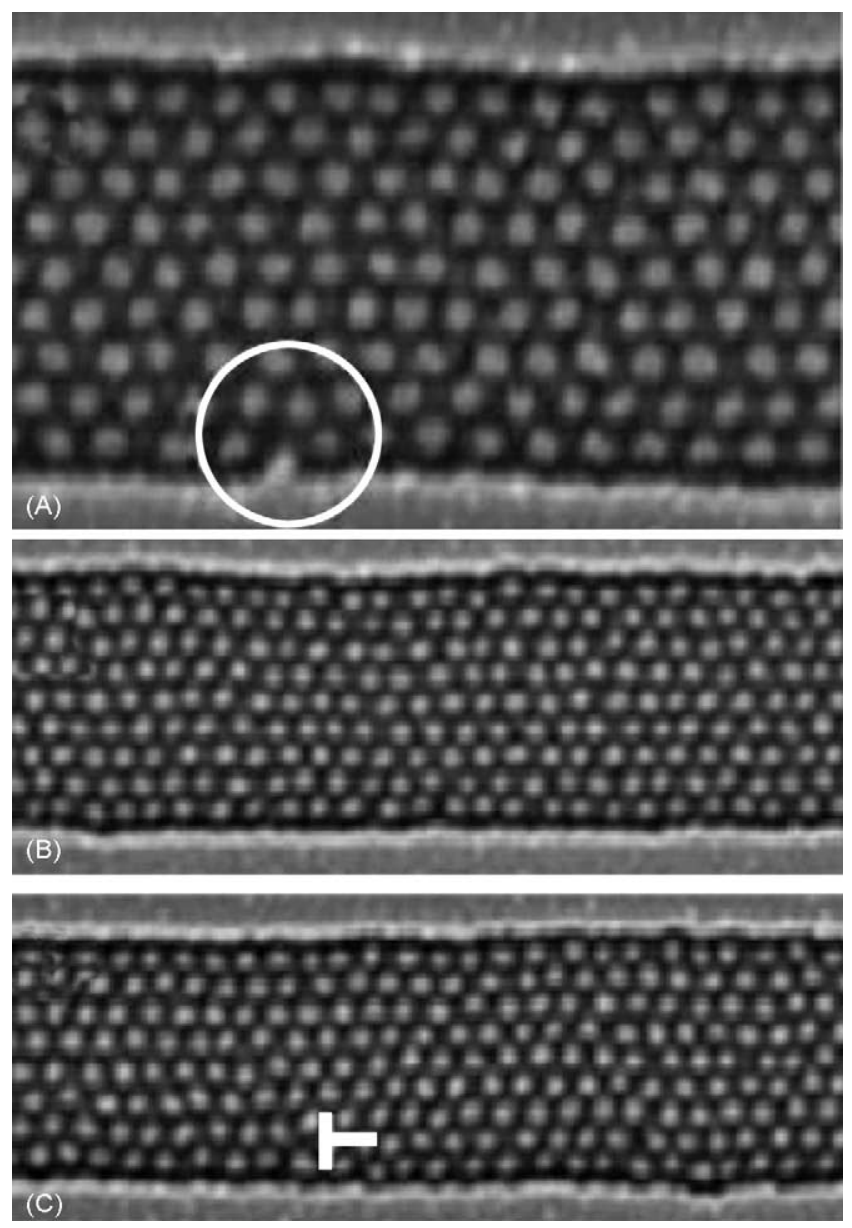

Fig. 12. The effect of substrate imperfections on graphoepitaxy. (A) A slight protrusion in the edge results in a point defect (a vacancy). Variations in the wall spacings may cause changes to the domain spacing (B) or dislocations as seen in (C). Reproduced with permission from Advanced Materials [17].

are crystallizable, then the melting temperatures of both materials are depressed. If the small molecule dissolves the block copolymer above its melting temperature and directionally crystallizes as it cools, it will act as a substrate on which thin films of the block copolymers are formed. In this case, two driving forces control the orientation of the crystallizable block copolymer: first, directional solidification and second, epitaxy as the nanodomains crystallize onto the preformed organic solvent crystal $[120,208]$. The combination of the two types of interactions determined the kinetically driven microstructures. In the case of an amorphous block copolymer, the fast directional solidification of the solvent during phase separation leads to an alignment of the block copolymer interface parallel to the fast growth direction of the solvent crystals. Both lamellar and cylindrical type nanodomains in a symmetric polystyrene-block-poly(methyl methacrylate) diblock copolymer and in an asymmetric polystyrene-block-polyisoprene, respectively, are globally aligned using either benzoic acid (BA) or anthracene (AN) as the crystallizable solvent $[208,209]$. The directional crystallization method can be combined with previously mentioned effects, particularly those related to film thickness, to control the orientation of the fast growth direction [210]. 

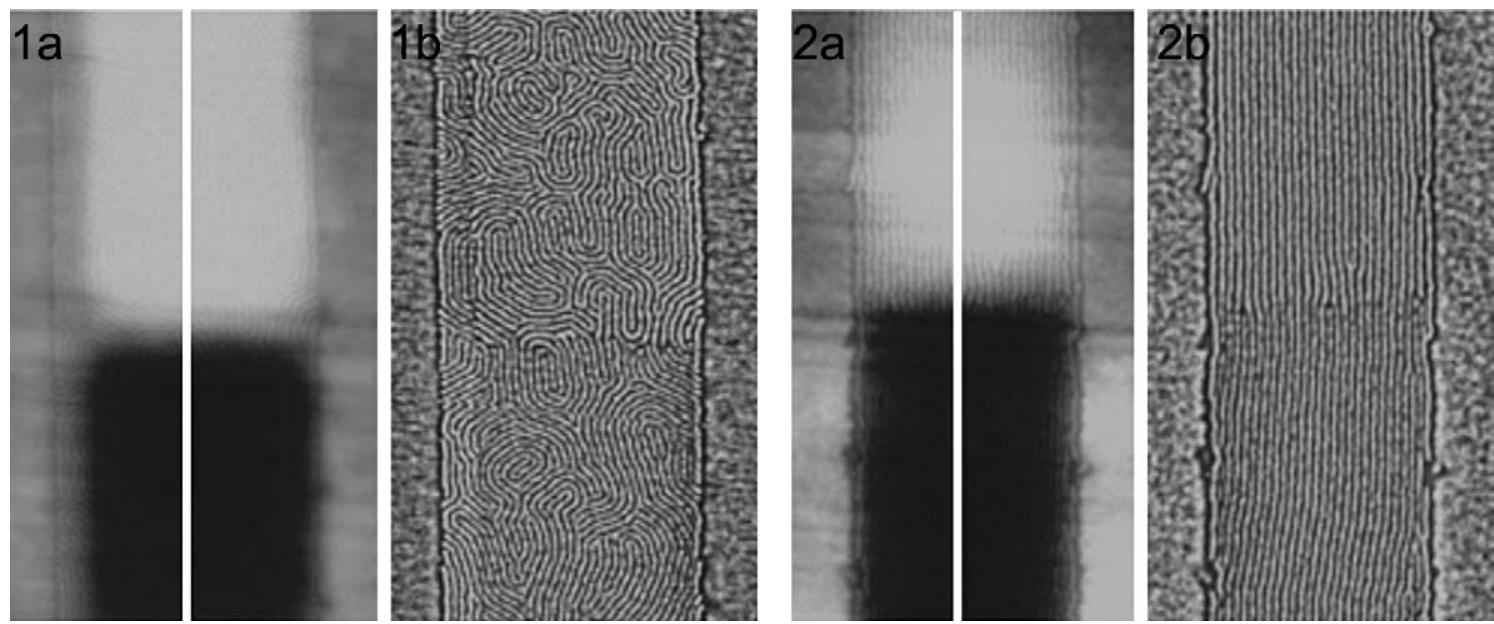

Fig. 13. Thin film of cylinder-forming PS-b-PI on a $35 \mathrm{~nm}$ deep grating. Tapping mode (a) topographic and (b) phase AFM images of the same region of the trough ( $960 \mathrm{~nm}$ wide) when the sample is annealed at $388 \mathrm{~K}$ for $2 \mathrm{~h}$ (1) and after further annealing at $403 \mathrm{~K}$ for $20 \mathrm{~h}$ (2). The bright and dark regions in the trough portion of the height image correspond to $5 \mathrm{~L} / 2$ - and $3 L / 2$-thick films. Initially, both thicknesses form very small grains of random orientation ( $1 \mathrm{~b}$ ) and then these grains reorient to form a single long grain with the axes of the cylinders aligned parallel to the channel edges. Reproduced with permission from Langmuir [204].

\subsection{Field-induced order}

Pioneering experiments by Amundson and coworkers showed that there is an electrostatic free energy penalty for interfaces perpendicular to an external field and as a result lamellae and cylinders tend to reorient parallel to the field [211-213]. The transition from parallel to perpendicular orientation is first order [214] and generally occurs when the electric field can overcome the parallel orientation induced by surface interactions [214,215]. Amundson and coworkers further inferred that defect motion is responsible for the reorientation of the domains from studying the structure of a bulk PSPMMA film with birefringence [211-213]. Krausch and coworkers propose both defect translation and grain rotation as mechanisms in block copolymer solutions resulting in similar reorientations [216,217]. Near the ODT, small-scale undulations allow for microdomain breakup and control reorganization, while in strongly segregated systems grain reorientation dominates [218,219]. In a block copolymer melt with cylindrical nanodomains, an electric field can not only induce a reorientation of nanodomains in an ordered but misaligned sample but also direct growth of nanodomains during formation at the ordering transition [220]. It was originally thought that the dielectric contrast between two blocks causes them to align so that their interfaces are parallel to an electric field and analogies to capacitors aligned in parallel seemed to capture the behavior of lamellae between electric fields [221]. However, Tsori et al. recently postulated that ions remaining in the film from the polymerization play a role in the reorientation process. Their calculations suggest that in many block copolymer systems, the relative strength of the orienting forces due to ion motion leads to a substantial reduction in the magnitude of the required external field [222].

In thin films, the electric field may be applied to orient the nanodomains either in the plane of the substrate or perpendicular to it. In-plane electrodes have been fabricated with standard optical lithography and electron-beam evaporation of a metal and used to orient long axes of cylinders of a PSb-PMMA system [223]. Initially, the axes of the cylinders are lying parallel to the substrate due to surface interactions. The axes can then be induced to stretch along the electric field lines when film is simultaneously heated above the glass transition temperature of both blocks. Since electric fields on 


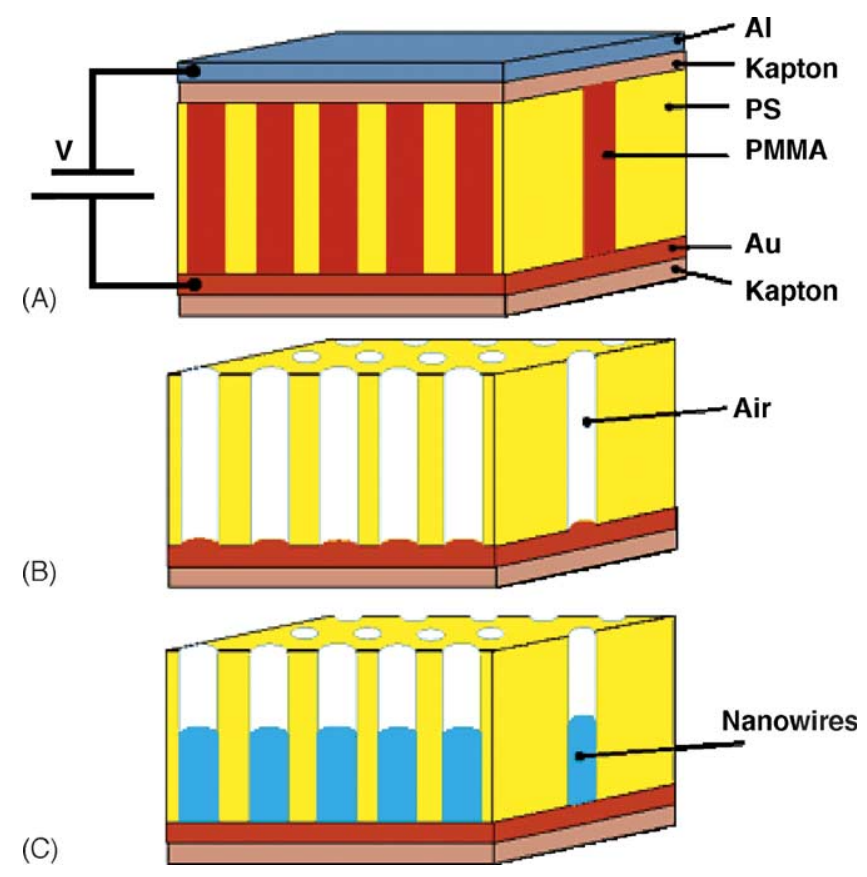

Fig. 14. A schematic representation of high-density nanowire fabrication in a polymer matrix. (A) An asymmetric diblock copolymer annealed above the glass transition temperature of the copolymer between two electrodes under an applied electric field, forming a hexagonal array of cylinders oriented normal to the film surface. (B) After removal of the minor component, a nanoporous film is formed. (C) By electrodeposition, nanowires can be grown in the porous template, forming an array of nanowires in a polymer matrix. Reproduced with permission from Science [224].

order $30 \mathrm{kV} / \mathrm{cm}$ were necessary to orient the domains, the patterning of an entire wafer would require the use of multiple electrodes and a careful understanding of the electric field formed when applying a voltage between electrodes on top of a thin insulating layer on a conducting substrate. Thurn-Albrecht et al. have shown that out-of-plane electrodes can force PS cylinders to orient perpendicular to the substrate and that these ordered arrays can be used to grow nanowires either by electrodeposition through the porous template or by using it as a mask for etching or patterning the underlying substrate, as schematically illustrated in Fig. 14 [224].

Analysis of scattering patterns indicated that during alignment of the lamellar nanodomains, the material goes through an intermediate state with substantially reduced long-range order. After orientation, the sample consists of many small grains with the lamellae being oriented parallel to the electric field and a random orientation in the plane perpendicular to the field [225].

Block copolymers in bulk are routinely aligned by shear fields. Cylinder-forming block copolymers which orient with their cylinder axes parallel to the flow direction have been organized into macroscopic single crystalline arrays [226]. Oscillatory shear performed between parallel plates allowed for control over film thickness, shear rate, and strain amplitude [227]. This form of bulk orientation has since been studied extensively but is beyond the scope of this review. Roll-casting, as developed by Albalak and Thomas, is one of the first techniques to translate shear induced orientation onto a film. In this case, the block copolymer is applied between two counter-rotating rolls and the complex shear field induced by the rolls orients the block copolymer as the solvent evaporates, eliminating the need for a high-temperature annealing step [228,229]. This technique has been used to align lamellae [229-231], cylinders [229,230], spheres [232], and bicontinuous nanodomains [233], but is only applicable to relatively thick films. Flow of a block copolymer solution down an inclined 
substrate can also orient thick films containing about 10 layers of cylinders [125]. A stamp made from an elastomeric mold pressed into a block copolymer solution on a solid substrate creates a flow field which has the potential for aligning the block copolymer [234]. Recently, Angelescu et al. have demonstrated that single layers of molten cylinders can be aligned by shear. In this technique, a simple, unpatterned PDMS stamp is placed on a heated film. When subjected to a lateral force, the stamp elastically distorts and shears the underlying block copolymer film which responds by reorienting in the direction of shear [235].

\subsection{Combination of techniques to yield three-dimensional control}

Many of the applications discussed previously in this article require three-dimensional order either for templating or to produce high aspect ratio, highly ordered structures such as lamellae and cylinders. Directional crystallization can be combined with a topographically patterned substrate to template a special variation in cylinder orientation, as shown in Fig. 15 [210]. Dark field TEM images (not shown here) indicate that the polyethylene lamellae are epitaxially crystallized onto the BA crystal and each cylindrical microdomain contains one crystalline polyethylene lamellae. The heteroepitaxy technique demonstrated by Nealey and coworkers induces lamellae in films just thicker than a lamellar spacing to stand upright and directly controls their long-range order simultaneously [195]. Simulations have demonstrated that cylinders on a hexagonally patterned substrate should also stand up and align [196].

True three-dimensional control over order frequently requires that more than one direction of organization be controlled usually by the application of multiple external fields. For instance, an

(A)
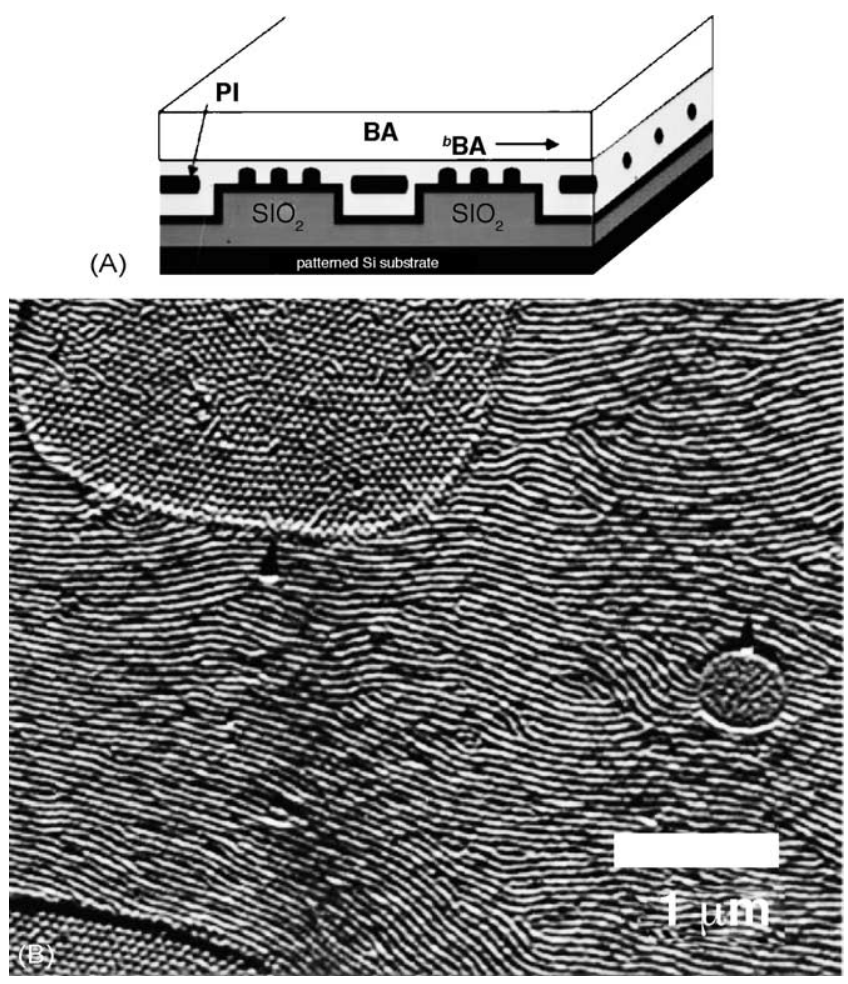

Fig. 15. Directional crystallization may be combined with a topographically patterned substrate to yield spatially varying orientations in block copolymer cylinders. Reproduced with permission from Applied Physics Letters [210]. 


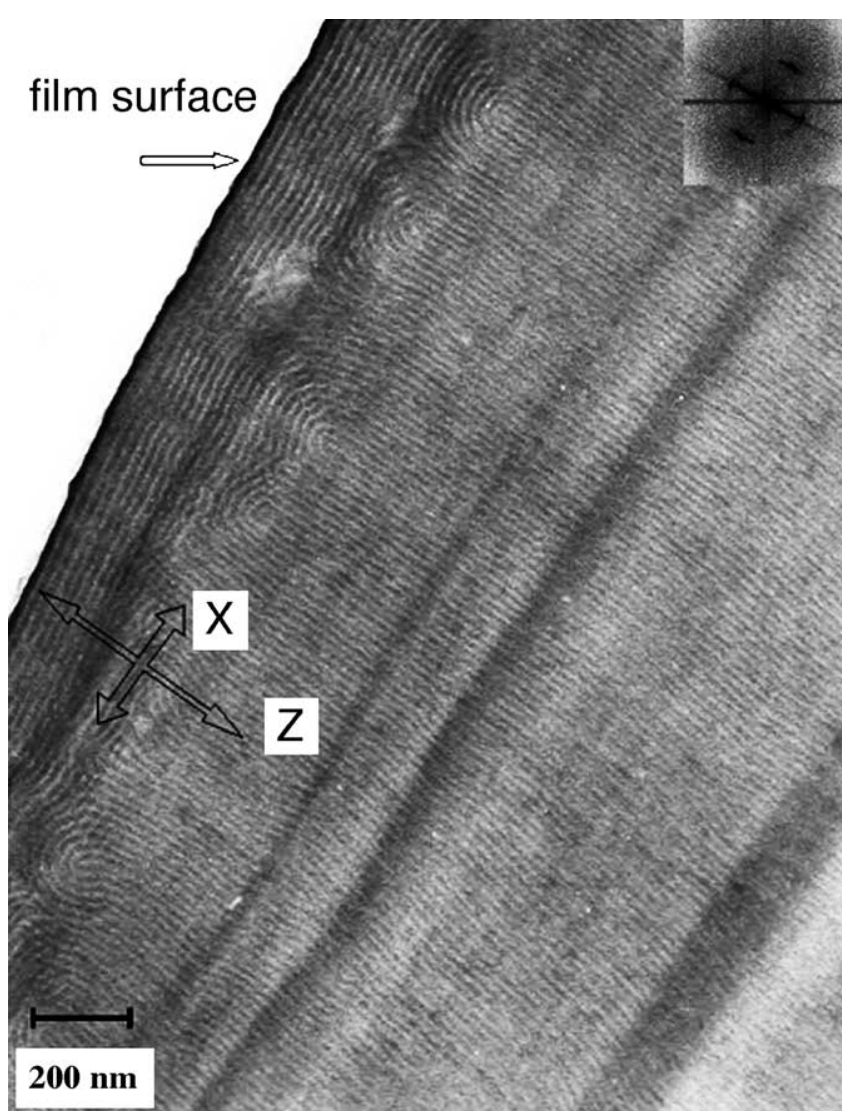

Fig. 16. Flow induced alignment along the $y$-direction and electric fields along the $z$-direction combine to create threedimensional order in this thin film. The top surface of the film is prevented from ordering due to surface effects. Reproduced with permission from Macromolecules [236].

elongational flow field applied to a lamellar film can lead to orientation of the lamellae normal to the surface of the film, though the lamellae seem to have very short persistence lengths within the plane of the film. Xu et al. then applied an electric field normal to the surface of the $30 \mu \mathrm{m}$ flow aligned film to further align the nanodomains [236] such that within the bulk of the film, there was near perfect alignment parallel to the flow direction and normal to the surface as seen in Fig. 16. The surfaces of the film, however, still demonstrate alignment parallel to the substrates due to surface effects. Alternatively, one could consider using a field alignment technique, such as an electric field or a surface modification to force cylinders or lamellae to align perpendicularly to a substrate in combination with topographic ordering techniques, such as graphoepitaxy to control order within the plane of the film. Li and Huck have used a surface neutralizing random copolymer in combination with nanoimprint graphoepitaxy to demonstrate control over both orientation and organization in cylindrical diblock copolymer films [206], though this technique has not yet been perfected to yield perfect crystals within perfectly straight channels.

\section{Conclusions and outlook}

External forces including interfacial energy, shear, and electromagnetic fields can direct the nanostructure of block copolymer arrays into technologically useful patterns. This review summarizes 
efforts to control block copolymer nanodomain morphology in thin films. Precise control over pattern formation has proven to be both challenging and successful in opening avenues to new applications. As is obvious throughout this review, countless new methods and interactions which could be used to gain greater levels of control are still not thoroughly investigated. For instance, true three-dimensional order requires control in multiple dimensions and this work has only just begun. Furthermore, while we can accurately control orientation and in some cases organization, we have not fully extended our understanding of bulk systems onto thin films. In particular, many thermal history effects have been exploited to maximize grain size in bulk samples yet have not been explored in thin films. Accessing the order-disorder transition and then quenching into the ordered regime yields extremely large grains in bulk $[144,145]$. Is this also true in thin films and if so, how does it affect the point defect density? The structure of two-dimensional films is strongly influenced by the surfaces sometimes resulting in surface reconstructions. We also do not yet understand exactly how these surface reconstructions relate to the bulk-type order of a thicker film. Spherical nanodomains in films containing only one layer of domains and the top layer of thicker films are known to organize into a hexagonal lattice, while in bulk they are arranged into a body-centered cubic array. The relationship between this top layer and those beneath it is unclear.

Two-dimensional systems present their own fundamental issues which we are just beginning to understand. We now know that two-dimensional spherical block copolymer arrays follow many of the predictions of classical two-dimensional crystals. The limit of quasi-long-range order in twodimensional crystals is fundamentally limited by nature of fluctuations in two dimensions. On one level, this puts a strict limit on the level of perfection one can expect from these potential lithographic masks, but it has also been demonstrated that heteroepitaxy may break this limit by forcing perfection. Will high degrees of topographical confinement do the same? Furthermore, questions are arising
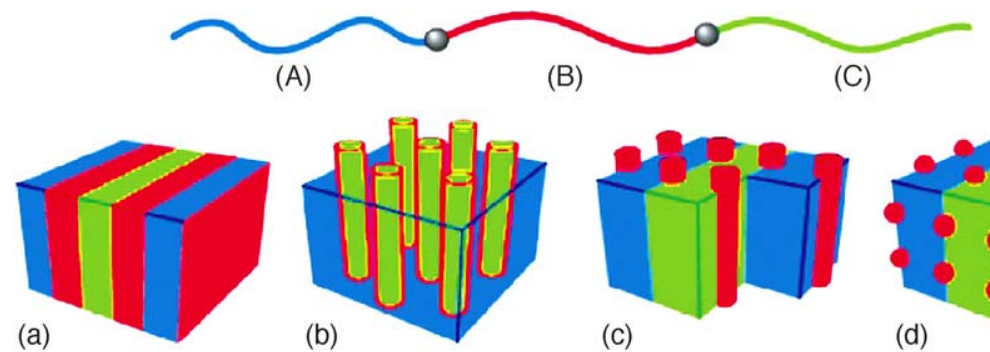

(c)
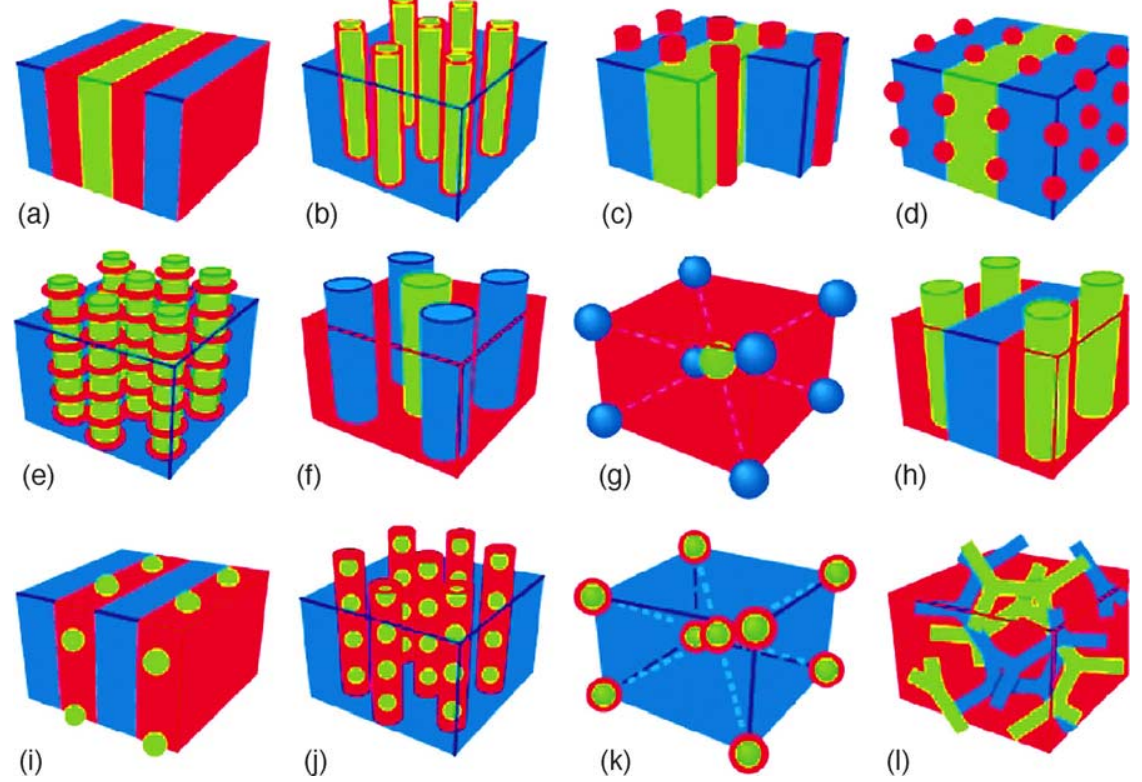

Fig. 17. Morphologies for linear $\mathrm{ABC}$ triblock copolymer. A combination of block sequences (ABC, ACB, BAC), composition and block molecular weights creates a large number of new morphologies. Reprinted with permission from Physics Today [2]. 
regarding the complexity of patterns available in block copolymer systems. The use of multiblock copolymers may allow for access more unusual architectures. ABC triblock copolymers in bulk have been observed to self-assemble into at least a dozen distinct morphologies, as shown in Fig. 17. A number of these morphologies have also been observed in thin films [126,237]. An alluring future direction of research involves nanopatterning with these complex architectures. A helical coil wrapped around a cylindrical nanodomain (Fig. 17e) has been observed in thin film structures [238] and in perpendicular orientation could yield very interesting resonator applications, while a cylinder in cylinder morphology, as shown in Fig. 17b might allow for the patterning of very small, high aspect ratio columns. The dual colored sphere morphology (Fig. 17g) could be useful in patterning dots on a square lattice not currently accessible via diblock copolymer lithography. The incorporation of a small amount of homopolymer has also been shown to have interesting effects on the nanopatterning of block copolymers [239-241]. As of yet, it is unclear how standard alignment techniques will work on these more complex systems.

A number of exciting potential applications have been discussed within this review including nanolithographic masks to pattern magnetic data storage and nanowires, the templating of nanoparticles, nanotubes, and nanoporous materials. Functional block copolymers are being explored for use in batteries, fuel cells, photonics, optoelectronic devices, and biological applications. It is apparent that our collective knowledge of self-assembly will soon extend into these much more complex, nonideal systems. Furthermore, our knowledge of block copolymer systems is now poised to tackle problems regarding the perfection of order and extending towards smaller length scales for nanolithography and larger ones for photonics.

\section{Acknowledgements}

RAS gratefully acknowledges the financial support of the U.S. Department of Energy Lawrence Berkeley Laboratories. Helpful discussions with Nitash Balsara and Ed Kramer are greatly appreciated.

\section{References}

[1] F.S. Bates, G.H. Fredrickson, Annu. Rev. Phys. Chem. 41 (1990) 525-557.

[2] F.S. Bates, G.H. Fredrickson, Phys. Today 52 (1999) 32-38.

[3] C. Harrison, J.A. Dagata, D.H. Adamson, Lithography with self-assembled block copolymer domains, in: I.W. Hamley (Ed.), Developments in Block Copolymer Science and Technology, Wiley, Chichester, West Sussex, UK/ Hoboken, NJ, 2004, , pp. 295-323.

[4] M. Park, C. Harrison, P.M. Chaikin, R.A. Register, D.H. Adamson, Science 276 (1997) 1401-1404.

[5] T. Xu, H.C. Kim, J. DeRouchey, C. Seney, C. Levesque, P. Martin, C.M. Stafford, T.P. Russell, Polymer 42 (2001) 9091-9095.

[6] K.W. Guarini, C.T. Black, K.R. Milkove, R.L. Sandstrom, J. Vac. Sci. Technol. B 19 (2001) 2784-2788.

[7] C.T. Black, K.W. Guarini, K.R. Milkove, S.M. Baker, T.P. Russell, M.T. Tuominen, Appl. Phys. Lett. 79 (2001) 409 411.

[8] K.W. Guarini, C.T. Black, Y. Zhang, H. Kim, E.M. Sikorski, I.V. Babich, J. Vac. Sci. Technol. B 20 (2002) $2788-2792$.

[9] M. Park, P.M. Chaikin, R.A. Register, D.H. Adamson, Appl. Phys. Lett. 79 (2001) 257-259.

[10] K. Shin, K.A. Leach, J.T. Goldbach, D.H. Kim, J.Y. Jho, M. Tuominen, C.J. Hawker, T.P. Russell, Nano Lett. 2 (2002) 933-936.

[11] E. Jeoung, T.H. Galow, J. Schotter, M. Bal, A. Ursache, M.T. Tuominen, C.M. Stafford, T.P. Russell, V.M. Rotello, Langmuir 17 (2001) 6396-6398.

[12] R.G.H. Lammertink, M.A. Hempenius, V.Z.H. Chan, E.L. Thomas, G.J. Vancso, Chem. Mater. 13 (2001) $429-434$. 
[13] J.Y. Cheng, C.A. Ross, V.Z.H. Chan, E.L. Thomas, R.G.H. Lammertink, G.J. Vancso, Adv. Mater. 13 (2001) $1174-$ 1178.

[14] S.B. Clendenning, I. Manners, Macromolecular Symposia 196 (2003) 71-76.

[15] W.Y. Chan, A.Y. Cheng, S.B. Clendenning, I. Manners, Macromol. Symp. 209 (2004) 163-176.

[16] J.Y. Cheng, C.A. Ross, E.L. Thomas, H.I. Smith, G.J. Vancso, Appl. Phys. Lett. 81 (2002) 3657-3659.

[17] J.Y. Cheng, C.A. Ross, E.L. Thomas, H.I. Smith, G.J. Vancso, Adv. Mater. 15 (2003) 1599-1602.

[18] K. Liu, S.M. Baker, M. Tuominen, T.P. Russell, I.K. Schuller, Phys. Rev. B 6305 (2001).

[19] K. Naito, H. Hieda, M. Sakurai, Y. Kamata, K. Asakawa, IEEE Trans. Magn. 38 (2002) 1949-1951.

[20] K. Asakawa, T. Hiraoka, H. Hieda, M. Sakurai, Y. Kamata, J. Photopolym. Sci. Technol. 15 (2002) 465-470.

[21] P. Du, M. Li, K. Douki, X. Li, C.B.W. Garcia, A. Jain, D.-M. Smilgies, L.J. Fetters, S.M. Gruner, U. Wiesner, C.K. Ober, Adv. Mater. 16 (2004) 953-957.

[22] D.H. Kim, Z.Q. Lin, H.C. Kim, U. Jeong, T.P. Russell, Adv. Mater. 15 (2003) 811-815.

[23] S.Y. Chou, P.R. Krauss, P.J. Renstrom, J. Vac. Sci. Technol. B 14 (1996) 4129-4133.

[24] D.J. Resnick, W.J. Dauksher, D. Mancini, K.J. Nordquist, T.C. Bailey, S. Johnson, N. Stacey, J.G. Ekerdt, C.G. Willson, S.V. Sreenivasan, N. Schumaker, J. Vac. Sci. Technol. B 21 (2003) 2624-2631.

[25] T.C. Bailey, S.C. Johnson, S.V. Sreenivasan, J.G. Ekerdt, C.G. Willson, D.J. Resnick, J. Photopolym. Sci. Technol. 15 (2002) 481-486.

[26] T.L. Morkved, P. Wiltzius, H.M. Jaeger, D.G. Grier, T.A. Witten, Appl. Phys. Lett. 64 (1994) $422-424$.

[27] H.C. Kim, X.Q. Jia, C.M. Stafford, D.H. Kim, T.J. McCarthy, M. Tuominen, C.J. Hawker, T.P. Russell, Adv. Mater. 13 (2001) 795-799.

[28] W.A. Lopes, H.M. Jaeger, Nature 414 (2001) 735-738.

[29] Y.N.C. Chan, R.R. Schrock, R.E. Cohen, J. Am. Chem. Soc. 114 (1992) 7295-7296.

[30] B.H. Sohn, R.E. Cohen, Acta Polym. 47 (1996) 340-343.

[31] J. Yue, V. Sankaran, R.E. Cohen, R.R. Schrock, J. Am. Chem. Soc. 115 (1993) 4409-4410.

[32] Y.N.C. Chan, G.S.W. Craig, R.R. Schrock, R.E. Cohen, Chem. Mater. 4 (1992) 885-894.

[33] R.E. Cohen, Curr. Opin. Solid State Mater. Sci. 4 (1999) 587-590.

[34] E. Sulman, V. Matveeva, A. Usanov, Y. Kosivtsov, G. Demidenko, L. Bronstein, D. Chernyshov, P. Valetsky, J. Mol. Catal. A: Chem. 146 (1999) 265-269.

[35] O.A. Platonova, L.M. Bronstein, S.P. Solodovnikov, I.M. Yanovskaya, E.S. Obolonkova, P.M. Valetsky, E. Wenz, M. Antonietti, Colloid Polym. Sci. 275 (1997) 426-431.

[36] L. Bronstein, D. Chernyshov, P. Valetsky, N. Tkachenko, H. Lemmetyinen, J. Hartmann, S. Forster, Langmuir 15 (1999) 83-91.

[37] M.V. Seregina, L.M. Bronstein, O.A. Platonova, D.M. Chernyshov, P.M. Valetsky, J. Hartmann, E. Wenz, M. Antonietti, Chem. Mater. 9 (1997) 923-931.

[38] S.R. Ahmed, S.B. Ogale, P. Kofinas, IEEE Trans. Magn. 39 (2003) 2198-2200.

[39] R.T. Clay, R.E. Cohen, Supramol. Sci. 4 (1997) 113-119.

[40] G.D. Brown, J.J. Watkins, Carbon dioxide dilated block copolymer templates for nanostructured materials, in: L. Merhari, L.T. Wille, K. Gonsalves, M.F. Gyure, S. Matsui, L.J. Whitman (Eds.), Materials Research Society Proceedings, vol. 584, 2000, pp. 169-174.

[41] B.H. Sohn, B.H. Seo, Chem. Mater. 13 (2001) 1752-1757.

[42] S. Horiuchi, T. Fujita, T. Hayakawa, Y. Nakao, Langmuir 19 (2003) 2963-2973.

[43] J. Raez, R. Barjovanu, J.A. Massey, M.A. Winnik, I. Manners, Angew. Chem. Int. Ed. 39 (2000) 3862-3874.

[44] J.A. Massey, M.A. Winnik, I. Manners, V.Z.H. Chan, J.M. Ostermann, R. Enchelmaier, J.P. Spatz, M. Moller, J. Am. Chem. Soc. 123 (2001) 3147-3148.

[45] S.B. Clendenning, S. Han, N. Coombs, C. Paquet, M.S. Rayat, D. Grozea, P.M. Brodersen, R.N.S. Sodhi, C.M. Yip, Z.H. Lu, I. Manners, Adv. Mater. 16 (2004) 291-295.

[46] J.P. Spatz, S. Mossmer, C. Hartmann, M. Moller, T. Herzog, M. Krieger, H.G. Boyen, P. Ziemann, B. Kabius, Langmuir 16 (2000) 407-415.

[47] Q. Fu, S.M. Huang, J. Liu, J. Phys. Chem. B 108 (2004) 6124-6129.

[48] R. Glass, M. Moller, J.P. Spatz, Nanotechnology 14 (2003) 1153-1160.

[49] B. Koslowski, S. Strobel, T. Herzog, B. Heinz, H.G. Boyen, R. Notz, P. Ziemann, J.P. Spatz, M. Moller, J. Appl. Phys. 87 (2000) 7533-7538.

[50] S.H. Lee, F.S. Diana, A. Badolato, P.M. Petroff, E.J. Kramer, J. Appl. Phys. 95 (2004) 5922-5924.

[51] A. Sidorenko, I. Tokarev, S. Minko, M. Stamm, J. Am. Chem. Soc. 125 (2003) 12211-12216.

[52] K. Almdal, K.A. Koppi, F.S. Bates, K. Mortensen, Macromolecules 25 (1992) 1743-1751. 
[53] F.S. Bates, M.F. Schulz, J.H. Rosedale, K. Almdal, Macromolecules 25 (1992) 5547-5550.

[54] F.S. Bates, G.H. Fredrickson, Macromolecules 27 (1994) 1065-1067.

[55] F.S. Bates, M.F. Schulz, A.K. Khandpur, S. Forster, J.H. Rosedale, K. Almdal, K. Mortensen, Faraday Discuss. (1994) 7-18.

[56] R. Krishnamoorti, W.W. Graessley, N.P. Balsara, D.J. Lohse, Macromolecules 27 (1994) 3073-3081.

[57] C.C. Lin, S.V. Jonnalagadda, P.K. Kesani, H.J. Dai, N.P. Balsara, Macromolecules 27 (1994) 7769-7780.

[58] W.W. Graessley, R. Krishnamoorti, G.C. Reichart, N.P. Balsara, L.J. Fetters, D.J. Lohse, Macromolecules 28 (1995) 1260-1270.

[59] H.S. Jeon, J.H. Lee, N.P. Balsara, Macromolecules 31 (1998) 3328-3339.

[60] K. Almdal, M.A. Hillmyer, F.S. Bates, Macromolecules 35 (2002) 7685-7691.

[61] J.T. Chen, E.L. Thomas, C.K. Ober, S.S. Hwang, Macromolecules 28 (1995) 1688-1697.

[62] A.N. Semenov, S.V. Vasilenko, Zh. Eksperimentalnoi I Teoreticheskoi Fiziki 90 (1986) 124-140.

[63] A. Halperin, Macromolecules 23 (1990) 2724-2731.

[64] C. Singh, M. Goulian, A.J. Liu, G.H. Fredrickson, Macromolecules 27 (1994) 2974-2986.

[65] M. Muller, M. Schick, Macromolecules 29 (1996) 8900-8903.

[66] M.W. Matsen, C. Barrett, J. Chem. Phys. 109 (1998) 4108-4118.

[67] W. Li, D. Gersappe, Macromolecules 34 (2001) 6783-6789.

[68] M. Reenders, G. ten Brinke, Macromolecules 35 (2002) 3266-3280.

[69] V. Pryamitsyn, V. Ganesan, J. Chem. Phys. 120 (2004) 5824-5838.

[70] J.T. Chen, E.L. Thomas, C.K. Ober, G.P. Mao, Science 273 (1996) 343-346.

[71] T.J. Deming, Adv. Drug Delivery Rev. 54 (2002) 1145-1155.

[72] H. Schlaad, M. Antonietti, Eur. Phys. J. E 10 (2003) 17-23.

[73] H. Kukula, H. Schlaad, M. Antonietti, S. Forster, J. Am. Chem. Soc. 124 (2002) 1658-1663.

[74] B.M. Discher, Y.Y. Won, D.S. Ege, J.C.M. Lee, F.S. Bates, D.E. Discher, D.A. Hammer, Science 284 (1999) 11431146.

[75] S. Lecommandoux, H.A. Klok, M. Sayar, S.I. Stupp, J. Polym. Sci. A: Polym. Chem. 41 (2003) 3501-3518.

[76] V. Breedveld, A.P. Nowak, J. Sato, T.J. Deming, D.J. Pine, Macromolecules 37 (2004) 3943-3953.

[77] E.G. Bellomo, M.D. Wyrsta, L. Pakstis, D.J. Pochan, T.J. Deming, Nat. Mater. 3 (2004) 244-248.

[78] J.J. Cheng, T.J. Deming, Macromolecules 34 (2001) 5169-5174.

[79] H. Schlaad, H. Kukula, B. Smarsly, M. Antonietti, T. Pakula, Polymer 43 (2002) 5321-5328.

[80] N.C. Greenham, S.C. Moratti, D.D.C. Bradley, R.H. Friend, A.B. Holmes, Nature 365 (1993) 628-630.

[81] Q.B. Pei, G. Yu, C. Zhang, Y. Yang, A.J. Heeger, Science 269 (1995) 1086-1088.

[82] B. de Boer, U. Stalmach, P.F. van Hutten, C. Melzer, V.V. Krasnikov, G. Hadziioannou, Polymer 42 (2001) 9097-9109.

[83] U. Stalmach, B. de Boer, C. Videlot, P.F. van Hutten, G. Hadziioannou, J. Am. Chem. Soc. 122 (2000) 5464-5472.

[84] G. Hadziioannou, MRS Bull. 27 (2002) 456-460.

[85] W.J. Li, H.B. Wang, L.P. Yu, T.L. Morkved, H.M. Jaeger, Macromolecules 32 (1999) 3034-3044.

[86] R. Lazzaroni, P. Leclere, A. Couturiaux, V. Parente, B. Francois, J.L. Bredas, Synth. Met. 102 (1999) 1279-1282.

[87] M.A. Hempenius, B.M.W. Langeveld-Voss, J. van Haare, R.A.J. Janssen, S.S. Sheiko, J.P. Spatz, M. Moller, E.W. Meijer, J. Am. Chem. Soc. 120 (1998) 2798-2804.

[88] G.N. Tew, M.U. Pralle, S.I. Stupp, J. Am. Chem. Soc. 121 (1999) 9852-9866.

[89] L.H. Radzilowski, B.O. Carragher, S.I. Stupp, Macromolecules 30 (1997) 2110-2119.

[90] S.H. Kim, M.J. Misner, T. Xu, M. Kimura, T.P. Russell, Adv. Mater. 16 (2004) 226-230.

[91] S.A. Jenekhe, X.L. Chen, Science 283 (1999) 372-375.

[92] F.M. Gray, J.R. Maccallum, C.A. Vincent, J.R.M. Giles, Macromolecules 21 (1988) 392-397.

[93] V. Chandrasekhar, Blockcopolym. Polyelectrolytes Biodegrad. 135 (1998) 139-205.

[94] P.P. Soo, B.Y. Huang, Y.I. Jang, Y.M. Chiang, D.R. Sadoway, A.M. Mayes, J. Electrochem. Soc. 146 (1999) $32-$ 37.

[95] T.H. Epps, T.S. Bailey, H.D. Pham, F.S. Bates, Chem. Mater. 14 (2002) 1706-1714.

[96] T.H. Epps, T.S. Bailey, R. Waletzko, F.S. Bates, Macromolecules 36 (2003) 2873-2881.

[97] S.C. Mui, P.E. Trapa, B. Huang, P.P. Soo, M.I. Lozow, T.C. Wang, R.E. Cohen, A.N. Mansour, S. Mukerjee, A.M. Mayes, D.R. Sadoway, J. Electrochem. Soc. 149 (2002) A1610-A1615.

[98] S.E. Bullock, P. Kofinas, Macromolecules 37 (2004) 1783-1786.

[99] S.E. Bullock, P. Kofinas, J. Power Sources 132 (2004) 256-260.

[100] H.B. Eitouni, N.P. Balsara, H. Hahn, J.A. Pople, M.A. Hempenius, Macromolecules 35 (2002) 7765-7772.

[101] J.Y. Cheng, C.A. Ross, E.L. Thomas, H.I. Smith, G.J. Vancso, Adv. Mater. 15 (2003) 1599-1603. 
[102] C. Hinderling, Y. Keles, T. Stockli, H.E. Knapp, T. de los Arcos, P. Oelhafen, I. Korczagin, M.A. Hempenius, G.J. Vancso, R.L. Pugin, H. Heinzelmann, Adv. Mater. 16 (2004) 876-879.

[103] G. Krausch, Mater. Sci. Eng. R: Rep. 14 (1995) 1-94.

[104] T.L. Morkved, W.A. Lopes, J. Hahm, S.J. Sibener, H.M. Jaeger, Polymer 39 (1998) 3871-3875.

[105] R. Magerle, Phys. Rev. Lett. 85 (2000) 2749-2752.

[106] C. Harrison, M. Park, P. Chaikin, R.A. Register, D.H. Adamson, N. Yao, Macromolecules 31 (1998) $2185-2189$.

[107] R.A. Segalman, A. Hexemer, R.C. Hayward, E.J. Kramer, Macromolecules 36 (2003) 3272-3288.

[108] C. Harrison, D.E. Angelescu, M. Trawick, Z.D. Cheng, D.A. Huse, P.M. Chaikin, D.A. Vega, J.M. Sebastian, R.A. Register, D.H. Adamson, Europhys. Lett. 67 (2004) 800-806.

[109] M.R. Hammond, S.W. Sides, G.H. Fredrickson, E.J. Kramer, J. Ruokolainen, S.F. Hahn, Macromolecules 36 (2003) 8712-8716.

[110] C. Harrison, Z.D. Cheng, S. Sethuraman, D.A. Huse, P.M. Chaikin, D.A. Vega, J.M. Sebastian, R.A. Register, D.H. Adamson, Phys. Rev. E 6601 (2002) U346-U372.

[111] R.A. Segalman, A. Hexemer, E.J. Kramer, Phys. Rev. Lett. 91 (2003).

[112] M.L. Trawick, M. Megens, C. Harrison, D.E. Angelescu, D.A. Vega, P.M. Chaikin, R.A. Register, D.H. Adamson, Scanning 25 (2003) 25-33.

[113] A. Hexemer, G.E. Stein, E.J. Kramer, Moiré patterns of 2D block copolymer arrays and their defects, in: Bulletin of the American Physical Society March Meeting, vol. 49, part I, Montreal, Canada, 2004, p. 232.

[114] D.E. Angelescu, C.K. Harrison, M.L. Trawick, P.M. Chaikin, R.A. Register, D.H. Adamson, Appl. Phys. A: Mater. Sci. Process. 78 (2004) 387-392.

[115] P. Muller-Buschbaum, R. Cubitt, W. Petry, Langmuir 19 (2003) 7778-7782.

[116] P. Muller-Buschbaum, J.S. Gutmann, R. Cubitt, W. Petry, Phys. B: Condens. Matter 350 (2004) 207-210.

[117] G. Kim, M. Libera, Macromolecules 31 (1998) 2569-2577.

[118] K.E. Strawhecker, S.K. Kumar, J.F. Douglas, A. Karim, Macromolecules 34 (2001) 4669-4672.

[119] I.W. Hamley, The Physics of Block Copolymers, Oxford University Press, New York, 1998, p. 425.

[120] C. De Rosa, C. Park, E.L. Thomas, B. Lotz, Nature 405 (2000) 433-437.

[121] R.J. Albalak, M.S. Capel, E.L. Thomas, Polymer 39 (1998) 1647-1656.

[122] H. Elbs, K. Fukunaga, R. Stadler, G. Sauer, R. Magerle, G. Krausch, Macromolecules 32 (1999) 1204-1211.

[123] T. Hashimoto, M. Shibayama, H. Kawai, Macromolecules 16 (1983) 1093-1101.

[124] T.P. Lodge, C. Pan, X. Jin, Z. Liu, J. Zhao, W.W. Maurer, F.S. Bates, J. Polym. Sci. B: Polym. Phys. 33 (1995) 22892293.

[125] M. Kimura, M.J. Misner, T. Xu, S.H. Kim, T.P. Russell, Langmuir 19 (2003) 9910-9913.

[126] H. Elbs, C. Drummer, V. Abetz, G. Krausch, Macromolecules 35 (2002) 5570-5577.

[127] K. Fukunaga, H. Elbs, R. Magerle, G. Krausch, Macromolecules 33 (2000) 947-953.

[128] S.J. Niu, R.F. Saraf, Macromolecules 36 (2003) 2428-2440.

[129] P. Alexandridis, R.J. Spontak, Curr. Opin. Colloid Interface Sci. 4 (1999) 130-139.

[130] V.S. RamachandraRao, R.R. Gupta, T.P. Russell, J.J. Watkins, Macromolecules 34 (2001) 7923-7925.

[131] B.D. Vogt, V.S. RamachandraRao, R.R. Gupta, K.A. Lavery, T.J. Francis, T.P. Russell, J.J. Watkins, Macromolecules 36 (2003) 4029-4036.

[132] G.H. Fredrickson, E. Helfand, J. Chem. Phys. 87 (1987) 697-705.

[133] G. Coulon, T.P. Russell, V.R. Deline, P.F. Green, Macromolecules 22 (1989) 2581-2589.

[134] T.P. Russell, G. Coulon, V.R. Deline, D.C. Miller, Macromolecules 22 (1989) 4600-4606.

[135] S.H. Anastasiadis, T.P. Russell, S.K. Satija, C.F. Majkrzak, Phys. Rev. Lett. 62 (1989) 1852-1855.

[136] A. Menelle, T.P. Russell, S.H. Anastasiadis, S.K. Satija, C.F. Majkrzak, Phys. Rev. Lett. 68 (1992) 67-70.

[137] M.D. Foster, M. Sikka, N. Singh, F.S. Bates, S.K. Satija, C.F. Majkrzak, J. Chem. Phys. 96 (1992) 8605-8615.

[138] A. Knoll, A. Horvat, K.S. Lyakhova, G. Krausch, G.J.A. Sevink, A.V. Zvelindovsky, R. Magerle, Phys. Rev. Lett. 8903 (2002) 035501.

[139] M.J. Fasolka, D.J. Harris, A.M. Mayes, M. Yoon, S.G.J. Mochrie, Phys. Rev. Lett. 79 (1997) 3018-3021.

[140] L. Rockford, Y. Liu, P. Mansky, T.P. Russell, M. Yoon, S.G.J. Mochrie, Phys. Rev. Lett. 82 (1999) $2602-2605$.

[141] J. Heier, E. Sivaniah, E.J. Kramer, Macromolecules 32 (1999) 9007-9012.

[142] R.A. Segalman, K.E. Schaefer, G.H. Fredrickson, E.J. Kramer, S. Magonov, Macromolecules 36 (2003) $4498-4506$.

[143] Z. Li, S. Qu, M.H. Rafailovich, J. Sokolov, M. Tolan, M.S. Turner, J. Wang, S.A. Schwarz, H. Lorenz, J.P. Kotthaus, Macromolecules 30 (1997) 8410-8419.

[144] H.J. Dai, N.P. Balsara, B.A. Garetz, M.C. Newstein, Phys. Rev. Lett. 77 (1996) 3677-3680.

[145] M.C. Newstein, B.A. Garetz, N.P. Balsara, M.Y. Chang, H.J. Dai, Macromolecules 31 (1998) 64-76. 
[146] W.G. Kim, B.A. Garetz, M.C. Newstein, N.P. Balsara, J. Polym. Sci. B: Polym. Phys. 39 (2001) 2231-2242.

[147] T. Hashimoto, J. Bodycomb, Y. Funaki, K. Kimishima, Macromolecules 32 (1999) 952-954.

[148] H. Yokoyama, T.E. Mates, E.J. Kramer, Macromolecules 33 (2000) 1888-1898.

[149] M.W. Hamersky, M.A. Hillmyer, M. Tirrell, F.S. Bates, T.P. Lodge, E.D. von Meerwall, Macromolecules 31 (1998) 5363-5370.

[150] M.W. Hamersky, M. Tirrell, T.P. Lodge, Langmuir 14 (1998) 6974-6979.

[151] H. Yokoyama, E.J. Kramer, Macromolecules 31 (1998) 7871-7876.

[152] T.P. Lodge, M.A. Blazey, Z. Liu, I.W. Hamley, Macromol. Chem. Phys. 198 (1997) 983-995.

[153] S. Joly, A. Raquois, F. Paris, B. Hamdoun, L. Auvray, D. Ausserre, Y. Gallot, Phys. Rev. Lett. 77 (1996) $4394-4397$.

[154] J. Heier, J. Genzer, E.J. Kramer, F.S. Bates, S. Walheim, G. Krausch, J. Chem. Phys. 111 (1999) 11101-11110.

[155] G. Coulon, D. Ausserre, T.P. Russell, Abstr. Pap. Am. Chem. Soc. 197 (1989), 198.

[156] G. Coulon, B. Collin, D. Ausserre, D. Chatenay, T.P. Russell, J. Phys. 51 (1990) 2801-2811.

[157] G. Coulon, D. Ausserre, T.P. Russell, J. Phys. 51 (1990) 777-786.

[158] G. Coulon, B. Collin, D. Chatenay, Y. Gallot, J. Phys. Ii 3 (1993) 697-717.

[159] B. Collin, D. Chatenay, G. Coulon, D. Ausserre, Y. Gallot, Macromolecules 25 (1992) 1621-1622.

[160] M. Maaloum, D. Ausserre, D. Chatenay, G. Coulon, Y. Gallot, Phys. Rev. Lett. 68 (1992) 1575-1578.

[161] P. Bassereau, D. Brodbreck, T.P. Russell, H.R. Brown, K.R. Shull, Phys. Rev. Lett. 71 (1993) 1716-1719.

[162] P. Bassereau, D. Brodbeck, T.P. Russell, H.R. Brown, K.R. Shull, Phys. Rev. Lett. 74 (1995) 4961.

[163] J. Heier, E.J. Kramer, J. Groenewold, G.H. Fredrickson, Macromolecules 33 (2000) 6060-6067.

[164] P.C.M. Grim, I.A. Nyrkova, A.N. Semenov, G. Tenbrinke, G. Hadziioannou, Macromolecules 28 (1995) $7501-7513$.

[165] H. Yokoyama, E.J. Kramer, D.A. Hajduk, F.S. Bates, Macromolecules 32 (1999) 3353-3359.

[166] C. Harrison, D.H. Adamson, Z.D. Cheng, J.M. Sebastian, S. Sethuraman, D.A. Huse, R.A. Register, P.M. Chaikin, Science 290 (2000) 1558-1560.

[167] D. Angelescu, C. Harrison, M. Trawick, R.A. Register, P.M. Chaikin, First order melting in a 2D diblock copolymer system, in: Bulletin of the American Physical Society March Meeting, vol. 49, part II, Montreal, Canada, 2004, p. 958.

[168] D. Angelescu, C. Harrison, M. Trawick, J. Sebastian, P.M. Chaikin, R.A. Register, D.H. Adamson, Melting microdomain patterns in a diblock copolymer thin film, in: Bulletin of the American Physical Society March Meeting, vol. 47, part II, Indianapolis, Indiana, 2002, p. 900.

[169] M. Ibanescu, Y. Fink, S. Fan, E.L. Thomas, J.D. Joannopoulos, Science 289 (2000) 415-419.

[170] Y. Fink, J.N. Winn, S.H. Fan, C.P. Chen, J. Michel, J.D. Joannopoulos, E.L. Thomas, Science 282 (1998) $1679-1682$.

[171] A. Urbas, R. Sharp, Y. Fink, E.L. Thomas, M. Xenidou, L.J. Fetters, Adv. Mater. 12 (2000) 812-814.

[172] T.P. Russell, P. Lambooy, G.J. Kellogg, A.M. Mayes, Physica B 213 (1995) 22-25.

[173] P. Lambooy, T.P. Russell, G.J. Kellogg, A.M. Mayes, P.D. Gallagher, S.K. Satija, Phys. Rev. Lett. 72 (1994) 2899 2902.

[174] N. Koneripalli, R. Levicky, F.S. Bates, J. Ankner, H. Kaiser, S.K. Satija, Langmuir 12 (1996) 6681-6690.

[175] G.J. Kellogg, D.G. Walton, A.M. Mayes, P. Lambooy, T.P. Russell, P.D. Gallagher, S.K. Satija, Phys. Rev. Lett. 76 (1996) 2503-2506.

[176] P. Mansky, P. Chaikin, E.L. Thomas, J. Mater. Sci. 30 (1995) 1987-1992.

[177] P. Mansky, Y. Liu, E. Huang, T.P. Russell, C. Hawker, Science 275 (1997) 1458-1460.

[178] P. Mansky, T.P. Russell, C.J. Hawker, J. Mays, D.C. Cook, S.K. Satija, Phys. Rev. Lett. 79 (1997) $237-240$.

[179] P. Mansky, T.P. Russell, C.J. Hawker, M. Pitsikalis, J. Mays, Macromolecules 30 (1997) 6810-6813.

[180] E. Huang, T.P. Russell, C. Harrison, P.M. Chaikin, R.A. Register, C.J. Hawker, J. Mays, Macromolecules 31 (1998) 7641-7650.

[181] J. Heier, E.J. Kramer, S. Walheim, G. Krausch, Macromolecules 30 (1997) 6610-6614.

[182] E. Sivaniah, Y. Hayashi, M. Iino, T. Hashimoto, K. Fukunaga, Macromolecules 36 (2003) 5894-5896.

[183] Y. Tsori, D. Andelman, Macromolecules 36 (2003) 8560-8566.

[184] J.W. Matthews, Epitaxial Growth, Academic Press, New York, 1975, p. 2v.

[185] H.I. Smith, D.C. Flanders, Appl. Phys. Lett. 32 (1978) 349-350.

[186] M.W. Geis, D.C. Flanders, H.I. Smith, J. Vac. Sci. Technol. 16 (1979) 1640-1643.

[187] E.I. Givargizov, N.N. Sheftal, V.I. Klykov, Diataxy (graphoepitaxy) and other approaches to oriented crystallization on amorphous substrates, Current Topics in Materials Science, vol. 10, North-Holland, Amsterdam/New York, 1982, pp. $3-53$.

[188] E.I. Givargizov, Thin Solid Films 189 (1990) 389-396.

[189] K.A. Mauritz, E. Baer, A.J. Hopfinger, Macromol. Rev. D: J. Polym. Sci. 13 (1978) 1-61. 
[190] C. De Rosa, C. Park, B. Lotz, J.C. Wittmann, L.J. Fetters, E.L. Thomas, Macromolecules 33 (2000) $4871-4876$.

[191] X.M. Yang, R.D. Peters, P.F. Nealey, H.H. Solak, F. Cerrina, Macromolecules 33 (2000) 9575-9582.

[192] T.K. Kim, X.M. Yang, R.D. Peters, B.H. Sohn, P.F. Nealey, J. Phys. Chem. B 104 (2000) 7403-7410.

[193] R.D. Peters, X.M. Yang, Q. Wang, J.J. de Pablo, P.F. Nealey, J. Vac. Sci. Technol. B 18 (2000) 3530-3534.

[194] X.M. Yang, R.D. Peters, P.F. Nealey, H.H. Solak, F. Cerrina, Macromolecules 33 (2000) 9575-9582.

[195] S.O. Kim, H.H. Solak, M.P. Stoykovich, N.J. Ferrier, J.J. de Pablo, P.F. Nealey, Nature 424 (2003) $411-414$.

[196] Q. Wang, P.F. Nealey, J.J. de Pablo, Macromolecules 36 (2003) 1731-1740.

[197] H. Chen, A. Chakrabarti, J. Chem. Phys. 108 (1998) 6897-6905.

[198] G.G. Pereira, D.R.M. Williams, Macromolecules 32 (1999) 758-764.

[199] Y. Tsori, D. Andelman, J. Chem. Phys. 115 (2001) 1970-1978.

[200] Y. Tsori, D. Andelman, Europhys. Lett. 53 (2001) 722-728.

[201] R.A. Segalman, H. Yokoyama, E.J. Kramer, Adv. Mater. 13 (2001) 1152-1155.

[202] C. Harrison, D.E. Angelescu, M. Trawick, Z.D. Cheng, D.A. Huse, P.M. Chaikin, D.A. Vega, J.M. Sebastian, R.A. Register, D.H. Adamson, Europlup. Lett. 67 (2004) 800-806.

[203] D. Sundrani, S.J. Sibener, Macromolecules 35 (2002) 8531-8539.

[204] D. Sundrani, S.B. Darling, S.J. Sibener, Langmuir 20 (2004) 5091-5099.

[205] D. Sundrani, S.B. Darling, S.J. Sibener, Nano Lett. 4 (2004) 273-276.

[206] H.W. Li, W.T.S. Huck, Nano Lett. 4 (2004) 1633-1636.

[207] M. Sakurai, H. Hiroyuki, Y. Kamata, A. Kikitsu, K. Naito, K. Asakawa, G. Adjanor, Nanoimprint-guided selfassembly of block copolymer films for the patterned media templates, in: APS March Meeting, vol. 48, part II, Austin, Texas, 2003, p. 534.

[208] C. Park, C. De Rosa, B. Lotz, L.J. Fetters, E.L. Thomas, Macromol. Chem. Phys. 204 (2003) 1514-1523.

[209] C. Park, C. De Rosa, E.L. Thomas, Macromolecules 34 (2001) 2602-2606.

[210] C. Park, J.Y. Cheng, M.J. Fasolka, A.M. Mayes, C.A. Ross, E.L. Thomas, C. De Rosa, Appl. Phys. Lett. 79 (2001) 848-850.

[211] K. Amundson, E. Helfand, Macromolecules 26 (1993) 1324-1332.

[212] K. Amundson, E. Helfand, X. Quan, S.D. Smith, Macromolecules 26 (1993) 2698-2703.

[213] K. Amundson, E. Helfand, X.N. Quan, S.D. Hudson, S.D. Smith, Macromolecules 27 (1994) 6559-6570.

[214] Y. Tsori, D. Andelman, Macromolecules 35 (2002) 5161-5170.

[215] T. Thurn-Albrecht, J. DeRouchey, T.P. Russell, H.M. Jaeger, Macromolecules 33 (2000) 3250-3253.

[216] A. Boker, H. Elbs, H. Hansel, A. Knoll, S. Ludwigs, H. Zettl, V. Urban, V. Abetz, A.H.E. Muller, G. Krausch, Phys. Rev. Lett. 8913 (2002) U102-U105.

[217] A. Boker, A. Knoll, H. Elbs, V. Abetz, A.H.E. Muller, G. Krausch, Macromolecules 35 (2002) 1319-1325.

[218] A.V. Kyrylyuk, A.V. Zvelindovsky, G.J.A. Sevink, J.G.E.M. Fraaije, Macromolecules 35 (2002) 1473-1476.

[219] A.V. Zvelindovsky, G.J.A. Sevink, Phys. Rev. Lett. 90 (2003).

[220] T. Thurn-Albrecht, J. DeRouchey, T.P. Russell, R. Kolb, Macromolecules 35 (2002) 8106-8110.

[221] G.G. Pereira, D.R.M. Williams, Macromolecules 32 (1999) 8115-8120.

[222] Y. Tsori, F. Tournilhac, L. Leibler, Macromolecules 36 (2003) 5873-5877.

[223] T.L. Morkved, M. Lu, A.M. Urbas, E.E. Ehrichs, H.M. Jaeger, P. Mansky, T.P. Russell, Science 273 (1996) 931-933.

[224] T. Thurn-Albrecht, J. Schotter, C.A. Kastle, N. Emley, T. Shibauchi, L. Krusin-Elbaum, K. Guarini, C.T. Black, M.T. Tuominen, T.P. Russell, Science 290 (2000) 2126-2129.

[225] J. DeRouchey, T. Thurn-Albrecht, T.P. Russell, R. Kolb, Macromolecules 37 (2004) 2538-2543.

[226] A. Keller, E. Pedemont, F.M. Willmout, Nature 225 (1970) 538.

[227] G. Hadziioannou, A. Mathis, A. Skoulios, Colloid Polym. Sci. 257 (1979) 136-139.

[228] R.J. Albalak, E.L. Thomas, J. Polym. Sci. B: Polym. Phys. 32 (1994) 341-350.

[229] R.J. Albalak, E.L. Thomas, J. Polym. Sci. B: Polym. Phys. 31 (1993) 37-46.

[230] R.J. Albalak, E.L. Thomas, M.S. Capel, Polymer 38 (1997) 3819-3825.

[231] Y. Cohen, R.J. Albalak, B.J. Dair, M.S. Capel, E.L. Thomas, Macromolecules 33 (2000) 6502-6516.

[232] E. Prasman, E.L. Thomas, J. Polym. Sci. B: Polym. Phys. 36 (1998) 1625-1636.

[233] B.J. Dair, A. Avgeropoulos, N. Hadjichristidis, M. Capel, E.L. Thomas, Polymer 41 (2000) 6231-6236.

[234] T. Deng, Y.H. Ha, J.Y. Cheng, C.A. Ross, E.L. Thomas, Langmuir 18 (2002) 6719-6722.

[235] D.E. Angelescu, J.H. Waller, D.H. Adamson, P. Deshpande, S.Y. Chou, R.A. Register, P.M. Chaikin, Adv. Mater. 16 (2004) 1736-1740.

[236] T. Xu, J.T. Goldbach, T.P. Russell, Macromolecules 36 (2003) 7296-7300.

[237] A. Boker, A.H.E. Muller, G. Krausch, Macromolecules 34 (2001) 7477-7488. 
[238] H. Elbs, V. Abetz, G. Hadziioannou, C. Drummer, G. Krausch, Macromolecules 34 (2001) 7917-7919.

[239] U. Jeong, D.Y. Ryu, D.H. Kho, J.K. Kim, J.T. Goldbach, D.H. Kim, T.P. Russell, Adv. Mater. 16 (2004) 533.

[240] U. Jeong, D.Y. Ryu, D.H. Kho, D.H. Lee, J.K. Kim, T.P. Russell, Macromolecules 36 (2003) 3626-3634.

[241] U.Y. Jeong, D.Y. Ryu, J.K. Kim, D.H. Kim, X.D. Wu, T.P. Russell, Macromolecules 36 (2003) 10126-10129.

[242] H. Yokoyama, E.J. Kramer, M.H. Rafailovich, J. Sokolov, S.A. Schwarz, Macromolecules 31 (1998) 8826-8830.

[243] J.Y. Cheng, A.M. Mayes, C.A. Ross, Nat. Mater. 3 (2004) 823-828. 